\title{
Simulation-Based Optimization of Markov Reward Processes
}

\author{
Peter Marbach and John N. Tsitsiklis, Fellow, IEEE
}

\begin{abstract}
This paper proposes a simulation-based algorithm for optimizing the average reward in a finite-state Markov reward process that depends on a set of parameters. As a special case, the method applies to Markov decision processes where optimization takes place within a parametrized set of policies. The algorithm relies on the regenerative structure of finite-state Markov processes, involves the simulation of a single sample path, and can be implemented online. A convergence result (with probability 1) is provided.
\end{abstract}

Index Terms-Markov reward processes, simulation-based optimization, stochastic approximation.

\section{INTRODUCTION}

$\mathbf{M}$ ARKOV decision processes, and the associated dynamic programming (DP) methodology [1], [25], provide a general framework for posing and analyzing problems of sequential decision making under uncertainty. DP methods rely on a suitably defined value function that has to be computed for every state in the state space. However, many interesting problems involve very large state spaces ("curse of dimensionality"). In addition, DP assumes the availability of an exact model, in the form of transition probabilities. In many practical situations, such a model is not available and one must resort to simulation or experimentation with an actual system. For all of these reasons, dynamic programming in its pure form, may be inapplicable.

The efforts to overcome the aforementioned difficulties involve the following two main ideas:

1) the use of simulation to estimate quantities of interest, thus avoiding model-based computations;

2) the use of parametric representations to overcome the curse of dimensionality.

Parametric representations, and the associated algorithms, can be broadly classified into three main categories.

1) Parametrized Value Functions: Instead of associating a value $V(i)$ with each state $i$, one uses a parametric form $\tilde{V}(i, r)$, where $r$ is a vector of tunable parameters

Manuscript received February 19, 1998; revised September 1, 1999. Recommended by Associate Editor D. Yao. This work was supported in part by Siemens AG, Munich, Germany, in part by Alcatel Bell, Belgium, and in part by the National Science Foundation under Contract DMI-9625489.

P. Marbach is with the Center for Communications Systems Research, University of Cambridge, Cambridge CB2 3DS, U.K. (e-mail: marbach@ccsr.cam.ac.uk).

J. N. Tsitsiklis is with the Laboratory for Information and Decision Systems, Massachusetts Institute of Technology, Cambridge, MA 02139 USA (e-mail jnt@mit.edu).

Publisher Item Identifier S 0018-9286(01)00327-0. (weights), and $\tilde{V}$ is a so-called approximation architecture. For example, $\tilde{V}(i, r)$ could be the output of a multilayer perceptron with weights $r$, when the input is $i$. Other representations are possible, e.g., involving polynomials, linear combinations of feature vectors, state aggregation, etc. When the main ideas from DP are combined with such parametric representations, one obtains methods that go under the names of "reinforcement learning" or "neuro-dynamic programming" (see [5] and [26] for textbook expositions, as well as the references therein). A key characteristic is that policy optimization is carried out in an indirect fashion; one tries to obtain a good approximation of the optimal value function of dynamic programming, and uses it to construct policies that are close to optimal. Such methods are reasonably well, though not fully, understood, and there have been some notable practical successes (see [5] and [26] for an overview), including the world-class backgammon player by Tesauro [28].

2) Parametrized Policies: In an alternative approach, which is the one considered in this paper, the tuning of a parametrized value function is bypassed. Instead, one considers a class of policies described in terms of a parameter vector $\theta$. Simulation is employed to estimate the gradient of the performance metric with respect to $\theta$, and the policy is improved by updating $\theta$ in a gradient direction. In some cases, the required gradient can be estimated using infinitesimal perturbation analysis (IPA) (see, e.g., [17], [12], [8], and the references therein). For general Markov processes, and in the absence of special structure, IPA is inapplicable, but gradient estimation is still possible using "likelihood-ratio" methods [14], [15], [13], [21], and [16].

3) Actor-Critic Methods: A third approach, which is a combination of the first two, includes parameterizations of the policy (actor) and of the value function (critic) [4]. While such methods seem particularly promising, theoretical understanding has been limited to the impractical case of lookup representations (one parameter per state) [19].

This paper concentrates on methods based on policy parameterization and (approximate) gradient improvement, in the spirit of item 2) above. While we are primarily interested in the case of Markov decision processes, almost everything applies to the more general case of Markov reward processes that depend on a parameter vector $\theta$, and we proceed within this broader context.

We start with a formula for the gradient of the performance metric that has been presented in different forms and for various contexts in [15], [7], [11], [18], [29], and [9]. We then suggest a 
method for estimating the terms that appear in that formula. This leads to a simulation-based method that updates the parameter vector $\theta$ at every regeneration time, in an approximate gradient direction. Furthermore, we show how to construct an online method that updates the parameter vector at each time step. The resulting method has some conceptual similarities with those described in [8] (that reference assumes, however, the availability of an IPA estimator, with certain guaranteed properties that are absent in our context) and in [18] (which, however, does not contain convergence results).

The method that we propose only keeps in memory and updates $2 K+1$ numbers, where $K$ is the dimension of $\theta$. Other than $\theta$ itself, this includes a vector similar to the "eligibility trace" in Sutton's temporal difference methods, and (as in [18]) an estimate $\tilde{\lambda}$ of the average reward under the current value of $\theta$. If that estimate was accurate, our method would be a standard stochastic gradient algorithm. However, as $\theta$ keeps changing, $\tilde{\lambda}$ is generally a biased estimate of the true average reward, and the mathematical structure of our method is more complex than that of stochastic gradient algorithms. For reasons that will become clearer later, standard approaches (e.g., martingale arguments or the ODE approach) do not seem to suffice for establishing convergence, and a more elaborate proof is necessary.

Our gradient estimator can also be derived or interpreted in terms of likelihood ratios [15], [13]. An alternative simulationbased stochastic gradient method, again based on a likelihood ratio formula, has been provided in [14], and uses the simulation of two regenerative cycles to construct an unbiased estimate of the gradient. We note some of the differences with the latter work. First, the methods in [14] involve a larger number of auxiliary quantities that are propagated in the course of a regenerative cycle. Second, our method admits a modification (see Sections IV and V) that can make it applicable even if the time until the next regeneration is excessive (in which case, likelihood ratio-based methods suffer from excessive variance). Third, our estimate $\tilde{\lambda}$ of the average reward is obtained as a (weighted) average of all past rewards (not just over the last regenerative cycle). In contrast, an approach such as the one in [14] would construct an independent estimate of $\tilde{\lambda}$ during each regenerative cycle, which should result in higher variance. Finally, our method brings forth and makes crucial use of the value (differential reward) function of dynamic programming. This is important because it paves the way for actor-critic methods in which the variance associated with the estimates of the differential rewards is potentially reduced by means of "learning" (value function approximation). Indeed, subsequent to the first writing of this paper, this latter approach has been pursued in [20], [27].

In summary, the main contributions of this paper are as follows.

1) We introduce a new algorithm for updating the parameters of a Markov reward process, on the basis of a single sample path. The parameter updates can take place either during visits to a certain recurrent state, or at every time step. We also specialize the method to Markov decision processes with parametrically represented policies. In this case, the method does not require the transition probabilities to be known.

2) We establish that the gradient (with respect to the parameter vector) of the performance metric converges to zero, with probability 1 , which is the strongest possible result for gradient-related stochastic approximation algorithms.

3) The method admits approximate variants with reduced variance, such as the one described in Section V, or various types of actor-critic methods.

The remainder of this paper is organized as follows. In Section II, we introduce our framework and assumptions, and state some background results, including a formula for the gradient of the performance metric. In Section III, we present an algorithm that performs updates during visits to a certain recurrent state, present our main convergence result, and provide a heuristic argument. Sections IV and V deal with variants of the algorithm that perform updates at every time step. In Section VI, we specialize our methods to the case of Markov decision processes that are optimized within a possibly restricted set of parametrically represented randomized policies. We present some numerical results in Section VII, and conclude in Section VIII. The lengthy proof of our main results is developed in Appendices I and II.

\section{Markov Reward Processes DePending on A PARAMETER}

In this section, we present our general framework, make a few assumptions, and state some basic results that will be needed later.

We consider a discrete-time, finite-state Markov chain $\left\{i_{n}\right\}$ with state space $S=\{1, \ldots, N\}$, whose transition probabilities depend on a parameter vector $\theta \in \Re^{K}$, and are denoted by

$$
p_{i j}(\theta)=P\left(i_{n}=j \mid i_{n-1}=i, \theta\right) .
$$

Whenever the state is equal to $i$, we receive a one-stage reward, that also depends on $\theta$, and is denoted by $g_{i}(\theta)$.

For every $\theta \in \Re^{K}$, let $P(\theta)$ be the stochastic matrix with entries $p_{i j}(\theta)$. Let $\mathcal{P}=\left\{P(\theta) \mid \theta \in \Re^{K}\right\}$ be the set of all such matrices, and let $\overline{\mathcal{P}}$ be its closure. Note that every element of $\overline{\mathcal{P}}$ is also a stochastic matrix and, therefore, defines a Markov chain on the same state space. We make the following assumptions.

Assumption 1: The Markov chain corresponding to every $P \in \overline{\mathcal{P}}$ is aperiodic. Furthermore, there exists a state $i^{*}$ which is recurrent for every such Markov chain.

We will often refer to the times that the state $i^{*}$ is visited as regeneration times.

Assumption 2: For every $i, j \in S$, the functions $p_{i j}(\theta)$ and $g_{i}(\theta)$ are bounded, twice differentiable, and have bounded first and second derivatives.

The performance metric that we use to compare different policies is the average reward criterion $\lambda(\theta)$, defined by

$$
\lambda(\theta)=\lim _{t \rightarrow \infty} \frac{1}{t} E_{\theta}\left[\sum_{k=0}^{t} g_{i_{k}}(\theta)\right] .
$$

Here, $i_{k}$ is the state at time $k$, and the notation $E_{\theta}[\cdot]$ indicates that the expectation is taken with respect to the distribution of the Markov chain with transition probabilities $p_{i j}(\theta)$. Under Assumption 1 , the average reward $\lambda(\theta)$ is well defined for every $\theta$, 
and does not depend on the initial state. Furthermore, the balance equations

$$
\begin{aligned}
\sum_{i=1}^{N} \pi_{i}(\theta) p_{i j}(\theta) & =\pi_{j}(\theta), \quad j=1, \ldots, N-1, \\
\sum_{i=1}^{N} \pi_{i}(\theta) & =1
\end{aligned}
$$

have a unique solution $\pi(\theta)=\left(\pi_{1}(\theta), \ldots, \pi_{N}(\theta)\right)$, with $\pi_{i}(\theta)$ being the steady-state probability of state $i$ under that particular value of $\theta$, and the average reward is equal to

$$
\lambda(\theta)=\sum_{i=1}^{N} \pi_{i}(\theta) g_{i}(\theta) .
$$

We observe that the balance equations (1) and (2) are of the form

$$
A(\theta) \pi(\theta)=a
$$

where $a$ is a fixed vector and $A(\theta)$ is an $N \times N$ matrix. (Throughout the paper, all vectors are treated as column vectors.) Using the fact that $A(\theta)$ depends smoothly on $\theta$, we have the following result.

Lemma 1: Let Assumptions 1 and 2 hold. Then, $\pi(\theta)$ and $\lambda(\theta)$ are twice differentiable, and have bounded first and second derivatives.

Proof: The balance equations are of the form $A(\theta) \pi(\theta)=$ $a$, where the entries of $A(\theta)$ have bounded second derivatives (Assumption 2). Since the balance equations have a unique solution, the matrix $A(\theta)$ is always invertible, and Cramer's rule yields

$$
\pi(\theta)=\frac{C(\theta)}{\operatorname{det}(A(\theta))}
$$

where $C(\theta)$ is a vector whose entries are polynomial functions of the entries of $A(\theta)$. Using Assumption 2, $C(\theta)$ and $\operatorname{det}(A(\theta))$ are twice differentiable and have bounded first and second derivatives.

More generally, suppose that $P \in \overline{\mathcal{P}}$, i.e., $P$ is the limit of the stochastic matrices $P\left(\theta_{k}\right)$ along some sequence $\theta_{k}$. The corresponding balance equations are again of the form $A(P) \pi=$ $a$, where $A(P)$ is a matrix depending on $P$. Under Assumption 1, these balance equations have again a unique solution, which implies that $|\operatorname{det}(A(P))|$ is strictly positive. Note that $|\operatorname{det}(A(P))|$ is a continuous function of $P$, and $P$ lies in the set $\overline{\mathcal{P}}$, which is closed and bounded. It follows that $|\operatorname{det}(A(P))|$ is bounded below by a positive constant $c$. Since every $P(\theta)$ belongs to $\overline{\mathcal{P}}$, it follows that $|\operatorname{det}(A(\theta))| \geq c>0$, for every $\theta$. This fact, together with (4), implies that $\pi(\theta)$ is twice differentiable and has bounded first and second derivatives. The same property holds true for $\lambda(\theta)$, as can be seen by twice differentiating the formula (3).

\section{A. The Gradient of $\lambda(\theta)$}

For any $\theta \in \Re^{K}$ and $i \in S$, we define the differential reward $v_{i}(\theta)$ of state $i$ by

$$
v_{i}(\theta)=E_{\theta}\left[\sum_{k=0}^{T-1}\left(g_{i_{k}}(\theta)-\lambda(\theta)\right) \mid i_{0}=i\right]
$$

where $i_{k}$ is the state at time $k$, and $T=\min \left\{k>0 \mid i_{k}=i^{*}\right\}$ is the first future time that state $i^{*}$ is visited. With this definition, it is well known that $v_{i^{*}}(\theta)=0$, and that the vector $v(\theta)=$ $\left(v_{1}(\theta), \ldots, v_{N}(\theta)\right)$ is a solution to the Poisson equation

$$
g(\theta)=v+\lambda(\theta) e-P(\theta) v
$$

where $g(\theta)=\left[g_{1}(\theta), \ldots, g_{N}(\theta)\right]$ and $e$ is equal to the all-one vector $(1, \ldots, 1)$.

The following proposition gives an expression for the gradient of the average reward $\lambda(\theta)$, with respect to $\theta$. A related expression (in a somewhat different context) was given in [18], and a proof can be found in [7]. (The latter reference does not consider the case where $g_{i}(\theta)$ depends on $\theta$, but the extension is immediate). Given the importance of this result, and because existing proofs are somewhat involved, we provide a concise self-contained proof, for the benefit of the reader.

Proposition 1: Let Assumptions 1 and 2 hold. Then,

$$
\nabla \lambda(\theta)=\sum_{i \in S} \pi_{i}(\theta)\left(\nabla g_{i}(\theta)+\sum_{j \in S} \nabla p_{i j}(\theta) v_{j}(\theta)\right) .
$$

Proof: We carry out the proof using vector notation, and using the superscript $T$ to denote vector transposition. All gradients are taken with respect to $\theta$, but to unclutter notation, the dependence on $\theta$ is suppressed.

We start with the Poisson equation $g=v+\lambda e-P v$, and left-multiply both sides with $\nabla \pi^{T}$, to obtain

$$
\left(\nabla \pi^{T}\right) g=\left(\nabla \pi^{T}\right) v+\lambda \nabla \pi^{T} e-\left(\nabla \pi^{T}\right)(P v) .
$$

Note that $\pi^{T} e=1$, which yields $\nabla \pi^{T} e=0$. Using the balance equation $\pi^{T} P=\pi^{T}$, we obtain

$$
\nabla \pi^{T}=\nabla\left(\pi^{T} P\right)=\left(\nabla \pi^{T}\right) P+\pi^{T}(\nabla P) .
$$

We right-multiply both sides by $v$, and use the resulting relation to rewrite the right-hand side of (6), leading to

$$
\left(\nabla \pi^{T}\right) g=\pi^{T}(\nabla P) v
$$

Thus,

$$
\nabla \lambda=\nabla\left(\pi^{T} g\right)=\pi^{T}(\nabla g)+\left(\nabla \pi^{T}\right) g=\pi^{T}(\nabla g)+\pi^{T}(\nabla P) v
$$

which is the desired result.

Equation (3) for $\lambda(\theta)$ suggests that $\nabla \lambda(\theta)$ could involve terms of the form $\nabla \pi_{i}(\theta)$, but the expression given by Proposition 1 involves no such terms. This property is very helpful in producing simulation-based estimates of $\nabla \lambda(\theta)$.

\section{B. An Idealized Gradient Algorithm}

Given that our goal is to maximize the average reward $\lambda(\theta)$, it is natural to consider gradient-type methods. If the gradient of $\lambda(\theta)$ could be computed exactly, we would consider a gradient algorithm of the form

$$
\theta_{k+1}=\theta_{k}+\gamma_{k} \nabla \lambda\left(\theta_{k}\right) .
$$

Based on the fact that $\lambda(\theta)$ has bounded second derivatives, and under suitable conditions on the stepsizes $\gamma_{k}$, it would follow that $\lim _{k \rightarrow \infty} \nabla \lambda\left(\theta_{k}\right)=0$ and that $\lambda\left(\theta_{k}\right)$ converges [2].

Alternatively, if we could use simulation to produce an unbiased estimate $h_{k}$ of $\nabla \lambda\left(\theta_{k}\right)$, we could then employ the stochastic gradient iteration

$$
\theta_{k+1}=\theta_{k}+\gamma_{k} h_{k} .
$$


The convergence of such a method can be established if we use a diminishing stepsize sequence and make suitable assumptions on the estimation errors. While one can construct unbiased estimates of the gradient [14], it does not appear possible to use them in an algorithm which updates the parameter vector $\theta$ at every time step-which is a desirable property, as discussed in Section III-D. This difficulty is bypassed by the method developed in Section III

\section{The Simulation-BASED Method}

In this section, we develop a simulation-based algorithm in which the gradient $\nabla \lambda(\theta)$ is replaced with a biased estimate, obtained by simulating a single sample path. We will eventually show that the bias asymptotically vanishes, which will then lead to a convergence result. For technical reasons, we make the following assumption on the transition probabilities $p_{i j}(\theta)$.

Assumption 3: For every $i$ and $j$, there exists a bounded function $L_{i j}(\theta)$ such that

$$
\nabla p_{i j}(\theta)=p_{i j}(\theta) L_{i j}(\theta), \quad \forall \theta .
$$

Note that when $p_{i j}(\theta)>0$, we have

$$
L_{i j}(\theta)=\frac{\nabla p_{i j}(\theta)}{p_{i j}(\theta)}
$$

which can be interpreted as a likelihood ratio derivative term [21]. Assumption 3 holds automatically if there exists a positive scalar $\epsilon$, such that for every $i, j \in S$, we have

$$
\text { either } p_{i j}(\theta)=0, \quad \forall \theta, \quad \text { or } \quad p_{i j}(\theta) \geq \epsilon \quad \forall \theta .
$$

\section{A. Estimation of $\nabla \lambda(\theta)$}

Throughout this subsection, we assume that the parameter vector $\theta$ is fixed to some value. Let $\left\{i_{n}\right\}$ be a sample path of the corresponding Markov chain, possibly obtained through simulation. Let $t_{m}$ be the time of the $m$ th visit at the recurrent state $i^{*}$. We refer to the sequence $i_{t_{m}}, i_{t_{m}+1}, \ldots, i_{t_{m+1}}$ as the $m$ th regenerative cycle, and we define its length $T_{m}$ by

$$
T_{m}=t_{m+1}-t_{m} .
$$

For a fixed $\theta$, the random variables $T_{m}$ are independent identically distributed, and have a (common) finite mean, denoted by $E_{\theta}[T]$.

Our first step is to rewrite the formula for $\nabla \lambda(\theta)$ in the form

$$
\nabla \lambda(\theta)=\sum_{i \in S} \pi_{i}(\theta)\left(\nabla g_{i}(\theta)+\sum_{j \in S} p_{i j}(\theta) L_{i j}(\theta) v_{j}(\theta)\right) \text {. }
$$

Estimating the term $\pi_{i}(\theta) \nabla g_{i}(\theta)$ through simulation is straightforward, assuming that we are able to compute $\nabla g_{i}(\theta)$ for any given $i$ and $\theta$. The other term can be viewed as the expectation of $v_{j}(\theta) L_{i j}(\theta)$, with respect to the steady-state probability $\pi_{i}(\theta) p_{i j}(\theta)$ of transitions from $i$ to $j$. Furthermore, the definition (5) of $v_{j}(\theta)$, suggests that if $t_{m}<n \leq t_{m+1}-1$, and $i_{n}=j$, we can use

$$
\tilde{v}_{i_{n}}(\theta, \tilde{\lambda})=\sum_{k=n}^{t_{m+1}-1}\left(g_{i_{k}}(\theta)-\tilde{\lambda}\right)
$$

to estimate $v_{j}(\theta)$, where $\tilde{\lambda}$ is some estimate of $\lambda(\theta)$. Note that $v_{i^{*}}(\theta)=0$ and does not need to be estimated. For this reason, we let

$$
\tilde{v}_{i_{n}}(\theta, \tilde{\lambda})=0, \quad \text { if } n=t_{m} .
$$

By accumulating the above described estimates over a regenerative cycle, we are finally led to an estimate of the direction of $\nabla \lambda(\theta)$ given by

$$
F_{m}(\theta, \tilde{\lambda})=\sum_{n=t_{m}}^{t_{m+1}-1}\left(\tilde{v}_{i_{n}}(\theta, \tilde{\lambda}) L_{i_{n-1} i_{n}}(\theta)+\nabla g_{i_{n}}(\theta)\right) \text {. }
$$

The random variables $F_{m}(\theta, \tilde{\lambda})$ are independent and identically distributed for different values of $m$, because the transitions during distinct regenerative cycles are independent.

We define $f(\theta, \tilde{\lambda})$ to be the expected value of $F_{m}(\theta, \tilde{\lambda})$, namely

$$
f(\theta, \tilde{\lambda})=E_{\theta}\left[F_{m}(\theta, \tilde{\lambda})\right] .
$$

The following proposition confirms that the expectation of $F_{m}(\theta, \tilde{\lambda})$ is aligned with $\nabla \lambda(\theta)$, to the extent that $\tilde{\lambda}$ is close to $\lambda(\theta)$.

Proposition 2: We have

$$
f(\theta, \tilde{\lambda})=E_{\theta}[T] \nabla \lambda(\theta)+G(\theta)(\lambda(\theta)-\tilde{\lambda})
$$

where

$$
G(\theta)=E_{\theta}\left[\sum_{n=t_{m}+1}^{t_{m+1}-1}\left(t_{m+1}-n\right) L_{i_{n-1} i_{n}}(\theta)\right] .
$$

Proof: Note that for $n=t_{m}+1, \ldots, t_{m+1}-1$, we have $\tilde{v}_{i_{n}}(\theta, \tilde{\lambda})=\sum_{k=n}^{t_{m+1}-1}\left(g_{i_{k}}(\theta)-\lambda(\theta)\right)+\left(t_{m+1}-n\right)(\lambda(\theta)-\tilde{\lambda})$. Therefore,

$$
\begin{aligned}
F_{m}(\theta, \tilde{\lambda})= & \sum_{n=t_{m}+1}^{t_{m+1}-1} a_{n} L_{i_{n-1} i_{n}}(\theta) \\
& +\sum_{n=t_{m}+1}^{t_{m+1}-1}\left(t_{m+1}-n\right)(\lambda(\theta)-\tilde{\lambda}) \\
& \cdot L_{i_{n-1} i_{n}}(\theta)+\sum_{n=t_{m}}^{t_{m+1}-1} \nabla g_{i_{n}}(\theta),
\end{aligned}
$$

where

$$
a_{n}=\sum_{k=n}^{t_{m+1}-1}\left(g_{i_{k}}(\theta)-\lambda(\theta)\right) .
$$

We consider separately the expectations of the three sums above. Using the definition of $G(\theta)$, the expectation of the second sum is equal to $G(\theta)(\lambda(\theta)-\tilde{\lambda})$. We then consider the third sum. As is well known, the expected sum of rewards over a regenerative cycle is equal to the steady-state expected reward times the expected length of the regenerative cycle. Therefore, the expectation of the third sum is

$$
E_{\theta}\left[\sum_{n=t_{m}}^{t_{m+1}-1} \nabla g_{i_{n}}(\theta)\right]=E_{\theta}[T] \sum_{i \in S} \pi_{i}(\theta) \nabla g_{i}(\theta) .
$$


We now focus on the expectation of the first sum. For $n=$ $t_{m}+1, \ldots, t_{m+1}-1$, let

$$
\Delta_{n}=\left(a_{n}-v_{i_{n}}(\theta)\right) L_{i_{n-1} i_{n}}(\theta) .
$$

Let $\mathcal{F}_{n}=\left\{i_{0}, \ldots, i_{n}\right\}$ stand for the history of the process up to time $n$. By comparing the definition (11) of $a_{n}$ with the definition (5) of $v_{i_{n}}(\theta)$, we obtain

$$
E_{\theta}\left[a_{n} \mid \mathcal{F}_{n}\right]=v_{i_{n}}(\theta) .
$$

It follows that $E_{\theta}\left[\Delta_{n} \mid \mathcal{F}_{n}\right]=0$

Let $\chi_{n}=1$ if $n<t_{m+1}$, and $\chi_{n}=0$, otherwise. For any $n>t_{m}$, we have

$$
\begin{aligned}
E_{\theta}\left[\chi_{n} \Delta_{n} \mid \mathcal{F}_{t_{m}}\right] & =E_{\theta}\left[E_{\theta}\left[\chi_{n} \Delta_{n} \mid \mathcal{F}_{n}\right] \mid \mathcal{F}_{t_{m}}\right] \\
& =E_{\theta}\left[\chi_{n} E_{\theta}\left[\Delta_{n} \mid \mathcal{F}_{n}\right] \mid \mathcal{F}_{t_{m}}\right]=0 .
\end{aligned}
$$

We then have

$$
\begin{aligned}
E_{\theta}\left[\sum_{n=t_{m}+1}^{t_{m+1}-1} \Delta_{n} \mid \mathcal{F}_{t_{m}}\right] & =E_{\theta}\left[\sum_{n=t_{m}+1}^{\infty} \chi_{n} \Delta_{n} \mid \mathcal{F}_{t_{m}}\right] \\
& =\sum_{n=t_{m}+1}^{\infty} E_{\theta}\left[\chi_{n} \Delta_{n} \mid \mathcal{F}_{t_{m}}\right]=0 .
\end{aligned}
$$

(The interchange of the summation and the expectation can be justified by appealing to the dominated convergence theorem.)

Therefore, we have

$$
\begin{aligned}
E_{\theta} & {\left[\sum_{n=t_{m}+1}^{t_{m+1}-1} a_{n} L_{i_{n-1} i_{n}}(\theta)\right] } \\
= & E_{\theta}\left[\sum_{n=t_{m}+1}^{t_{m+1}-1} v_{i_{n}}(\theta) L_{i_{n-1} i_{n}}(\theta)\right] .
\end{aligned}
$$

The right-hand side can be viewed as the total reward over a regenerative cycle of a Markov reward process, where the reward associated with a transition from $i$ to $j$ is $v_{j}(\theta) L_{i j}(\theta)$. Recalling that any particular transition has steady-state probability $\pi_{i}(\theta) p_{i j}(\theta)$ of being from $i$ to $j$, we obtain

$$
\begin{aligned}
& E_{\theta}\left[\sum_{n=t_{m}+1}^{t_{m+1}-1} a_{n} L_{i_{n-1} i_{n}}(\theta)\right] \\
& =E_{\theta}[T] \sum_{i \in S} \sum_{j \in S} \pi_{i}(\theta) p_{i j}(\theta) L_{i j}(\theta) v_{j}(\theta) .
\end{aligned}
$$

By combining (12) and (14), and comparing with the formula for $\nabla \lambda(\theta)$, we see that the desired result has been proved.

\section{B. An Algorithm that Updates at Visits to the Recurrent State}

We now use the approximate gradient direction provided by Proposition 2, and propose a simulation-based algorithm that performs updates at visits to the recurrent state $i^{*}$. We use the variable $m$ to index the times when the recurrent state $i^{*}$ is visited, and the corresponding updates. The form of the algorithm is the following. At the time $t_{m}$ that state $i^{*}$ is visited for the $m$ th time, we have available a current vector $\theta_{m}$ and an average reward estimate $\tilde{\lambda}_{m}$. We then simulate the process according to the transition probabilities $p_{i j}\left(\theta_{m}\right)$ until the next time $t_{m+1}$ that $i^{*}$ is visited, and update according to

$$
\begin{aligned}
& \theta_{m+1}=\theta_{m}+\gamma_{m} F_{m}\left(\theta_{m}, \tilde{\lambda}_{m}\right) \\
& \tilde{\lambda}_{m+1}=\tilde{\lambda}_{m}+\eta \gamma_{m} \sum_{n=t_{m}}^{t_{m+1}-1}\left(g_{i_{n}}\left(\theta_{m}\right)-\tilde{\lambda}_{m}\right)
\end{aligned}
$$

where $\gamma_{m}$ is a positive stepsize sequence (cf. Assumption 4) and $\eta>0$ allows to scale the stepsize for updating $\tilde{\lambda}$ by a positive constant. To see the rationale behind (16), note that the expected update direction for $\tilde{\lambda}$ is

$$
E_{\theta}\left[\sum_{n=t_{m}}^{t_{m+1}-1}\left(g_{i_{n}}(\theta)-\tilde{\lambda}\right)\right]=E_{\theta}[T](\lambda(\theta)-\tilde{\lambda})
$$

which moves $\tilde{\lambda}$ closer to $\lambda(\theta)$.

Assumption 4: The stepsizes $\gamma_{m}$ are nonnegative and satisfy

$$
\sum_{m=1}^{\infty} \gamma_{m}=\infty, \quad \sum_{m=1}^{\infty} \gamma_{m}^{2}<\infty .
$$

Assumption 4 is satisfied, for example, if we let $\gamma_{m}=1 / \mathrm{m}$. It can be shown that if $\theta$ is held fixed, but $\tilde{\lambda}$ keeps being updated according to (16), then $\tilde{\lambda}$ converges to $\lambda(\theta)$. However, if $\theta$ is also updated according to (15), then the estimate $\tilde{\lambda}_{m}$ can "lag behind" $\lambda\left(\theta_{m}\right)$. As a consequence, the expected update direction for $\theta$ will not be aligned with the gradient $\nabla \lambda(\theta)$.

An alternative approach that we do not pursue is to use different stepsizes for updating $\tilde{\lambda}$ and $\theta$. If the stepsize used to update $\theta$ is, in the limit, much smaller than the stepsize used to update $\tilde{\lambda}$, the algorithm exhibits a two-time scale behavior of the form studied in [3]. In the limit, $\tilde{\lambda}_{m}$ is an increasingly accurate estimate of $\lambda\left(\theta_{m}\right)$, and the algorithm is effectively a stochastic gradient algorithm. However, such a method would make slower progress, as far as $\theta$ is concerned. Our convergence results indicate that this alternative approach is not necessary.

We can now state our main result, which is proved in Appendix I.

Proposition 3: Let Assumptions 1-4 hold, and let $\left\{\theta_{m}\right\}$ be the sequence of parameter vectors generated by the above described algorithm. Then, $\lambda\left(\theta_{m}\right)$ converges and

$$
\lim _{m \rightarrow \infty} \nabla \lambda\left(\theta_{m}\right)=0
$$

with probability 1 .

\section{A Heuristic Argument}

In this subsection, we approximate the algorithm by a suitable ODE (as in [22]), and establish the convergence properties of the ODE. While this argument does not constitute a proof, it illustrates the rationale behind our convergence result.

We replace the update directions by their expectations under the current value of $\theta$. The resulting deterministic update equations take the form

$$
\begin{aligned}
& \theta_{m+1}^{d}=\theta_{m}^{d}+\gamma_{m} f\left(\theta_{m}^{d}, \tilde{\lambda}_{m}^{d}\right) \\
& \tilde{\lambda}_{m+1}^{d}=\tilde{\lambda}_{m}^{d}+\eta \gamma_{m} E_{\theta_{m}^{d}}[T]\left(\lambda\left(\theta_{m}^{d}\right)-\tilde{\lambda}_{m}^{d}\right)
\end{aligned}
$$


where $f(\theta, \tilde{\lambda})$ is given by Proposition 2 , and where $\theta_{m}^{d}$ and $\tilde{\lambda}_{m}^{d}$ are the deterministic counterparts of $\theta_{m}$ and $\tilde{\lambda}_{m}$, respectively. With an asymptotically vanishing stepsize, and after rescaling time, this deterministic iteration behaves similar to the following system of differential equations:

$$
\begin{aligned}
& \dot{\theta}_{t}=\nabla \lambda\left(\theta_{t}\right)+\frac{G\left(\theta_{t}\right)}{E_{\theta_{t}}[T]}\left(\lambda\left(\theta_{t}\right)-\tilde{\lambda}_{t}\right) \\
& \dot{\tilde{\lambda}}_{t}=\eta\left(\lambda\left(\theta_{t}\right)-\tilde{\lambda}_{t}\right) .
\end{aligned}
$$

Note that $\tilde{\lambda}_{t}$ and $\lambda\left(\theta_{t}\right)$ are bounded functions since the one-stage reward $g_{i}(\theta)$ is finite-valued and, therefore, bounded. We will now argue that $\tilde{\lambda}_{t}$ converges.

We first consider the case where the initial conditions satisfy $\tilde{\lambda}_{0} \leq \lambda\left(\theta_{0}\right)$. We then claim that

$$
\tilde{\lambda}_{t} \leq \lambda\left(\theta_{t}\right) \quad \forall t>0 .
$$

Indeed, suppose that at some time $t_{0}$ we have $\tilde{\lambda}_{t_{0}}=\lambda\left(\theta_{t_{0}}\right)$. If $\nabla \lambda\left(\theta_{t_{0}}\right)=0$, then we are at an equilibrium point of the differential equations, and we have $\tilde{\lambda}_{t}=\lambda\left(\theta_{t}\right)$ for all subsequent times. Otherwise, if $\nabla \lambda\left(\theta_{t_{0}}\right) \neq 0$, then $\dot{\theta}_{t_{0}}=\nabla \lambda\left(\theta_{t_{0}}\right)$, and $\dot{\lambda}\left(\theta_{t_{0}}\right)>0$. At the same time, we have $\dot{\tilde{\lambda}}_{t_{0}}=0$, and this implies that $\tilde{\lambda}_{t}<\lambda\left(\theta_{t}\right)$ for $t$ slightly larger than $t_{0}$. The validity of the claim (20) follows. As long as $\tilde{\lambda}_{t} \leq \lambda\left(\theta_{t}\right), \tilde{\lambda}_{t}$ is nondecreasing and since it is bounded, it must converge.

Suppose now that the initial conditions satisfy $\tilde{\lambda}_{0}>\lambda\left(\theta_{0}\right)$. As long as this condition remains true, $\tilde{\lambda}_{t}$ is nonincreasing. There are two possibilities. If this condition remains true for all times, then $\tilde{\lambda}_{t}$ converges. If not, then there exists a time $t_{0}$ such that $\tilde{\lambda}_{t_{0}}=\lambda\left(\theta_{t_{0}}\right)$, which takes us back to the previously considered case.

Having concluded that $\tilde{\lambda}_{t}$ converges, we can use (19) to argue that $\lambda\left(\theta_{t}\right)$ must also converge to the same limit. Then, in the limit, $\theta_{t}$ evolves according to $\dot{\theta}_{t}=\nabla \lambda\left(\theta_{t}\right)$, from which it follows that $\nabla \lambda\left(\theta_{t}\right)$ must go to zero.

We now comment on the nature of a rigorous proof. There are two common approaches for proving the convergence of stochastic approximation methods. One method relies on the existence of a suitable Lyapunov function and a martingale argument. In our context, $\lambda(\theta)$ could play such a role. However, as long as $\tilde{\lambda}_{m} \neq \lambda\left(\theta_{m}\right)$, our method cannot be expressed as a stochastic gradient algorithm and this approach does not go through. (Furthermore, it is unclear whether another Lyapunov function would do.) The second proof method, the so-called ODE approach, shows that the trajectories followed by the algorithm converge to the trajectories of a corresponding deterministic ODE, e.g., the ODE given by (18) and (19). This line of analysis generally requires the iterates to be bounded functions of time. In our case, such a boundedness property is not guaranteed to hold. For example, if $\theta$ stands for the weights of a neural network, it is certainly possible that certain "neurons" asymptotically saturate, and the corresponding weights drift to infinity. We therefore need a line of argument specially tailored to our particular algorithm. In rough terms, it proceeds along the same lines as the above provided deterministic analysis, except that we must also ensure that the stochastic terms are not significant.

\section{Implementation Issues}

For systems involving a large state space, as is the case in many applications, the interval between visits to the state $i^{*}$ can be large. Consequently,

1) the parameter vector $\theta$ gets updated only infrequently;

2) the estimate $F_{m}(\theta)$ can have a large variance.

In the following, we will address these two issues and propose two modified versions: one which updates $\theta$ at every time step, and one which reduces the variance of the updates.

\section{An Algorithm that Updates AT EVERy Time SteP}

In this section, we develop an algorithm which updates the parameter vector $\theta$ at every time step. We start by indicating an economical way of computing the update direction $F_{m}(\theta, \tilde{\lambda})$. This will allow us to break $F_{m}(\theta)$ into a sum of incremental updates carried out at each time step.

Taking into account that $\tilde{v}_{i_{t_{m}}}(\theta, \tilde{\lambda})=0,(8)$ becomes

$$
\begin{aligned}
F_{m}(\theta, \tilde{\lambda}) & \sum_{n=t_{m}+1}^{t_{m+1}-1} \tilde{v}_{i_{n}}(\theta, \tilde{\lambda}) L_{i_{n-1} i_{n}}(\theta)+\sum_{n=t_{m}}^{t_{m+1}-1} \nabla g_{i_{n}}(\theta) \\
= & \sum_{n=t_{m}+1}^{t_{m+1}-1}\left(\nabla g_{i_{n}}(\theta)+L_{i_{n-1} i_{n}}(\theta) \sum_{k=n}^{t_{m+1}-1}\left(g_{i_{k}}(\theta)-\tilde{\lambda}\right)\right) \\
& +\nabla g_{i^{\star}}(\theta) \\
= & \sum_{k=t_{m}+1}^{t_{m+1}-1}\left(\nabla g_{i_{k}}(\theta)+\left(g_{i_{k}}(\theta)-\tilde{\lambda}\right) \sum_{n=t_{m}+1}^{k} L_{i_{n-1} i_{n}}(\theta)\right) \\
& +\nabla g_{i^{\star}}(\theta) \\
= & \nabla g_{i^{*}}(\theta)+\sum_{k=t_{m}+1}^{t_{m+1}-1}\left(\nabla g_{i_{k}}(\theta)+\left(g_{i_{k}}(\theta)-\tilde{\lambda}\right) z_{k}\right)
\end{aligned}
$$

where, for $k=t_{m}+1, \ldots, t_{m+1}-1$,

$$
z_{k}=\sum_{n=t_{m}+1}^{k} L_{i_{n-1} i_{n}}(\theta)=\sum_{n=t_{m}+1}^{k} \frac{\nabla p_{i_{n-1} i_{n}}(\theta)}{p_{i_{n-1} i_{n}}(\theta)}
$$

is a vector (of the same dimension as $\theta$ ) that becomes available at time $k$. It can be updated recursively, with

$$
z_{t_{m}}=0
$$

and

$$
z_{k+1}=z_{k}+L_{i_{k} i_{k+1}}(\theta), \quad k=t_{m}, \ldots, t_{m+1}-2 .
$$

We note that $z_{k}$ is the likelihood ratio derivative that commonly arises in likelihood ratio gradient estimation [15], [13].

The preceding formulas suggest the following algorithm which updates $\theta$ at every time step. At a typical time $k$, the state is $i_{k}$, and the values of $\theta_{k}, z_{k}$, and $\tilde{\lambda}_{k}$ are available from the previous iteration. We update $\theta$ and $\tilde{\lambda}$ according to

$$
\begin{aligned}
& \theta_{k+1}=\theta_{k}+\gamma_{k}\left(\nabla g_{i_{k}}\left(\theta_{k}\right)+\left(g_{i_{k}}\left(\theta_{k}\right)-\tilde{\lambda}_{k}\right) z_{k}\right) \\
& \tilde{\lambda}_{k+1}=\tilde{\lambda}_{k}+\eta \gamma_{k}\left(g_{i_{k}}\left(\theta_{k}\right)-\tilde{\lambda}_{k}\right) .
\end{aligned}
$$


We then simulate a transition to the next state $i_{k+1}$ according to the transition probabilities $p_{i j}\left(\theta_{k+1}\right)$, and finally update $z$ by letting

$$
z_{k+1}= \begin{cases}0, & \text { if } i_{k+1}=i^{*} \\ z_{k}+L_{i_{k} i_{k+1}}\left(\theta_{k}\right), & \text { otherwise }\end{cases}
$$

In order to implement the algorithm, on the basis of the above equations, we only need to maintain in memory $2 K+1$ scalars, namely $\tilde{\lambda}$, and the vectors $\theta, z$.

To prove convergence of this version of the algorithm, we have to strengthen Assumption 1 of Section II. Assumption 1 states that for every fixed parameter $\theta$, we will eventually reach the state $i^{*}$. Here, we need to make sure that this will remain so, even if $\theta$ keeps changing; see [23] for further discussion of this assumption.

Assumption 5: There exist a state $i^{*} \in S$ and a positive integer $N_{0}$, such that, for every state $i \in S$ and every collection $\left\{P_{1}, \ldots, P_{N_{0}}\right\}$ of $N_{0}$ matrices in the set $\overline{\mathcal{P}}$, we have

$$
\sum_{n=1}^{N_{0}}\left[\prod_{l=1}^{n} P_{l}\right]_{i i^{*}}>0 .
$$

We also impose an additional condition on the stepsizes.

Assumption 6: The stepsizes $\gamma_{k}$ are nonincreasing. Furthermore, there exists a positive integer $p$ and a positive scalar $A$ such that

$$
\sum_{k=n}^{n+t}\left(\gamma_{n}-\gamma_{k}\right) \leq A t^{p} \gamma_{n}^{2} \quad \forall n, t>0 .
$$

Assumption 6 is satisfied, for example, if we let $\gamma_{k}=1 / k$. With this choice, and if we initialize $\tilde{\lambda}$ to zero, it is easily verified that $\tilde{\lambda}_{k}$ is equal to the average reward obtained in the first $k$ transitions.

We have the following convergence result, which is proved in Appendix II.

Proposition 4: Let Assumptions 1-6 hold, and let $\left\{\theta_{k}\right\}$ be the sequence of parameter vectors generated by the above described algorithm. Then, $\lambda\left(\theta_{k}\right)$ converges and

$$
\lim _{k \rightarrow \infty} \nabla \lambda\left(\theta_{k}\right)=0
$$

with probability 1 .

The algorithm of this section is similar to the algorithm of the preceding one, except that $\theta$ is continually updated in the course of a regenerative cycle. Because of the diminishing stepsize, these incremental updates are asymptotically negligible, and the difference between the two algorithms is inconsequential. Given that the algorithm of the preceding section converges, Proposition 4 is hardly surprising. The technique in our convergence proof use is similar to the one in [8]. However, mapped into the context of parametrized Markov reward processes, [8] assumes that the transition probabilities $p_{i j}(\theta)$ are independent of $\theta$ [the one-stage rewards $g_{i}(\theta)$ can still depend on $\theta$ ]. The situation here is more general, and a separate proof is needed.

\section{An Algorithm that may Reduce the Variance}

When the length of a regeneration cycle is large, the vector $z_{k}$ will also become large before it is reset to zero, resulting in high variance for the updates. (This is a generic difficulty associated with likelihood ratio methods.) For this reason, it may be preferable to introduce a forgetting factor $\alpha \in(0,1)$ and update $z_{k}$ according to

$$
z_{k+1}= \begin{cases}0, & \text { if } i_{k+1}=i^{*} \\ \alpha z_{k}+L_{i_{k} i_{k+1}}\left(\theta_{k}\right) & \text { otherwise. }\end{cases}
$$

This modification, which resembles the algorithm introduced in [18], can reduce the variance of a typical update, but introduces a new bias in the update direction. Given that gradient-type methods are fairly robust with respect to small biases, this modification may result in improved practical performance; see the numerical results in Section VII.

Similar to [18], this modified algorithm can be justified if we approximate the differential reward with

$$
v_{i}(\theta) \approx E_{\theta}\left[\sum_{k=0}^{T} \alpha^{k}\left(g_{i_{k}}(\theta)-\lambda(\theta)\right) \mid i_{0}=i\right]
$$

where $T=\min \left\{k>0 \mid i_{k}=i^{*}\right\}$ (which is increasingly accurate as $\alpha \uparrow 1$ ), use the estimate

$$
\tilde{v}_{i_{n}}(\theta, \tilde{\lambda})=\sum_{k=n}^{T} \alpha^{k}\left(g_{i_{k}}(\theta)-\tilde{\lambda}\right)
$$

instead of (7), and then argue similar to Section III. The analysis of this algorithm is carried out in [23] and, with less detail, in [24].

\section{Markov Decision PROCESSES}

In this section, we indicate how to apply our methodology to Markov decision processes. An important feature, which is evident from the formulas provided at the end of this section, is that the algorithm is "model-free": as long as the process can be simulated or is available for observation, explicit knowledge of the transition probabilities $p_{i j}(\theta)$ is not needed.

We consider a Markov decision processes [1], [25] with finite state space $S=\{1, \ldots, N\}$ and finite action space $U=$ $\{1, \cdots, M\}$. At any state $i$, the choice of a control action $u \in U$ determines the probability $p_{i j}(u)$ that the next state is $j$. The immediate reward at each time step is of the form $g_{i}(u)$, where $i$ and $u$ is the current state and action, respectively.

A (randomized) policy is defined as a mapping

$$
\mu: S \mapsto[0,1]^{M}
$$

with components $\mu_{u}(i)$ such that

$$
\sum_{u \in U} \mu_{u}(i)=1 \quad \forall i \in S .
$$

Under a policy $\mu$, and whenever the state is equal to $i$, action $u$ is chosen with probability $\mu_{u}(i)$, independent of everything else. If for every state $i$ there exists a single $u$ for which $\mu_{u}(i)$ is positive (and, therefore, unity), we say that we have a pure policy.

For problems involving very large state spaces, it is impossible to even describe an arbitrary pure policy $\mu$, since this requires a listing of the actions corresponding to each state. This leads us to consider policies described in terms of a parameter vector $\theta=\left(\theta_{1}, \cdots, \theta_{K}\right)$, whose dimension $K$ is tractable 
small. We are interested in a method that performs small incremental updates of the parameter $\theta$. A method of this type can work only if the policy has a smooth dependence on $\theta$, and this is the main reason why we choose to work with randomized policies.

We allow $\theta$ to be an arbitrary element of $\Re^{K}$. With every $\theta \in \Re^{K}$, we associate a randomized policy $\mu(\theta)$, which at any given state $i$ chooses action $u$ with probability $\mu_{u}(i, \theta)$. Naturally, we require that every $\mu_{u}(i, \theta)$ be nonnegative and that $\sum_{u \in U} \mu_{u}(i, \theta)=1$. Note that the resulting transition probabilities are given by

$$
p_{i j}(\theta)=\sum_{u \in U} \mu_{u}(i, \theta) p_{i j}(u)
$$

and the expected reward per stage is given by

$$
g_{i}(\theta)=\sum_{u \in U} \mu_{u}(i, \theta) g_{i}(u) .
$$

The objective is to maximize the average reward under policy $\mu(\theta)$, which is denoted by $\lambda(\theta)$. This is a special case of the framework of Section II. We now discuss the various assumptions introduced in earlier sections.

In order to satisfy Assumption 1, it suffices to assume that there exists a state $i^{*}$ which is recurrent under every pure policy, a property which is satisfied in many interesting problems. In order to satisfy Assumption 2, it suffices to assume that the policy has a smooth dependence on $\theta$; in particular, that $\mu_{u}(i, \theta)$ is twice differentiable (in $\theta$ ) and has bounded first and second derivatives. Finally, Assumption 3 is implied by the following condition.

Assumption 7: For every $i$ and $u$, there exists a bounded function $L_{u}(i, \theta)$ such that

$$
\nabla \mu_{u}(i, \theta)=\mu_{u}(i, \theta) L_{u}(i, \theta) \quad \forall \theta .
$$

This assumption can be satisfied in any of the following ways.

1) Consider a smoothly parametrized function $r_{u}(i, \theta)$ that maps state-action pairs $(i, u)$ to real numbers, and suppose that

$$
\mu_{u}(i, \theta)=\frac{\exp \left(r_{u}(i, \theta)\right)}{\sum_{v} \exp \left(r_{v}(i, \theta)\right)} .
$$

Assumption 7 is satisfied once we assume that $r_{u}(i, \theta)$ has bounded first and second derivatives. This particular form is common in the neural network literature: the $r_{u}(i, \theta)$ are the outputs of a neural network with input $(i, u)$ and internal weights $\theta$, and an action $u$ is selected by a randomized "soft maximum."

2) We may artificially restrict to policies for which there exists some $\epsilon>0$ such that

$$
\mu_{u}(i, \theta) \geq \epsilon \quad \forall i, u, \theta .
$$

Such policies introduce a minimal amount of "exploration," and ensure that every action will be tried infinitely often. This can be beneficial because the available experience with simulation-based methods for Markov decision processes indicates that performance can substantially degrade in the absence of exploration: a method may stall within a poor set of policies for the simple reason that the actions corresponding to better policies have not been sufficiently explored.

Since $\sum_{u \in U} \mu_{u}(i, \theta)=1$ for every $\theta$, we have $\sum_{u \in U} \nabla \mu_{u}(i, \theta)=0$, and

$$
\nabla g_{i}(\theta)=\sum_{u \in U} \nabla \mu_{u}(i, \theta)\left(g_{i}(u)-\lambda(\theta)\right) .
$$

Furthermore,

$$
\sum_{j \in S} \nabla p_{i j}(\theta) v_{j}(\theta)=\sum_{j \in S} \sum_{u \in U} \nabla \mu_{u}(i, \theta) p_{i j}(u) v_{j}(\theta) .
$$

Using these relations in the formula for $\nabla \lambda(\theta)$ provided by Proposition 1, and after some rearranging, we obtain

$$
\nabla \lambda(\theta)=\sum_{i \in S} \sum_{u \in U} \pi_{i}(\theta) \mu_{u}(i, \theta) q_{i, u}(\theta) \frac{\nabla \mu_{u}(i, \theta)}{\mu_{u}(i, \theta)}
$$

where

$$
\begin{aligned}
q_{i, u}(\theta) & =\left(g_{i}(u)-\lambda(\theta)\right)+\sum_{j \in S} p_{i j}(u) v_{j}(\theta) \\
& =E_{\theta}\left[\sum_{k=0}^{T-1}\left(g_{i_{k}}\left(u_{k}\right)-\lambda(\theta)\right) \mid i_{0}=i, u_{0}=u\right]
\end{aligned}
$$

and where $i_{k}$ and $u_{k}$ is the state and control at time $k$. Thus, $q_{i, u}(\theta)$ is the differential reward if control action $u$ is first applied in state $i$, and policy $\mu(\theta)$ is followed thereafter. It is the same as Watkins' $Q$-factor [30], suitably modified for the average reward case.

From here on, we can proceed as in Section III and obtain an algorithm that updates $\theta$ at the times $t_{m}$ that state $i^{*}$ is visited. The form of the algorithm is

$$
\begin{aligned}
& \theta_{m+1}=\theta_{m}+\gamma_{m} F_{m}\left(\theta_{m}, \tilde{\lambda}_{m}\right), \\
& \tilde{\lambda}_{m+1}=\tilde{\lambda}_{m}+\eta \gamma_{m} \sum_{n=t_{m}}^{t_{m+1}-1}\left(g_{i_{n}}\left(u_{n}\right)-\tilde{\lambda}_{m}\right)
\end{aligned}
$$

where

$$
F_{m}\left(\theta_{m}, \tilde{\lambda}_{m}\right)=\sum_{n=t_{m}}^{t_{m+1}-1} \tilde{q}_{i_{n}}, u_{n} \frac{\nabla \mu_{u_{n}}\left(i_{n}, \theta_{m}\right)}{\mu_{u_{n}}\left(i_{n}, \theta_{m}\right)},
$$

and

$$
\tilde{q}_{i_{n}}, u_{n}=\sum_{k=n}^{t_{m+1}-1}\left(g_{i_{k}}\left(u_{k}\right)-\tilde{\lambda}_{m}\right) .
$$

Similar to Section IV, an on-line version of the algorithm is also possible. The convergence results of Sections III and IV remain valid, with only notation changes in the proof.

\section{EXPERIMENTAL RESULTS FOR AN ADMISSION CONTROL PROBLEM}

In this section, we describe some numerical experiments with a call admission control problem. This problem arises when a service provider with limited resources (bandwidth) has to accept or reject incoming calls of several types, while taking into account current congestion. The objective is to maximize long-term average revenue. More details on the experiments reported here can be found in [23]. 


\section{A. Problem Formulation}

Consider a communication link with a total bandwidth of $B$ units, which supports a finite set $\{1,2, \ldots, M\}$ of different service types. Each service type is characterized by its bandwidth requirement $b(m)$, its call arrival rate $\alpha(m)$, and its average holding time $1 / \beta(m)$, where we assume that the calls (customers) arrive according to independent Poisson processes, and that the holding times are exponentially (and independently) distributed. When a new customer requests a connection, we can decide to reject, or, if enough bandwidth is available, to accept the customer. Once accepted, a customer of class $m$ seizes $b(m)$ units of bandwidth for the duration of the call. Whenever a call of service type $m$ gets accepted, we receive an immediate reward of $c(m)$ units. The reward $c(m)$ can be interpreted as the price customers of service type $m$ are paying for using $b(m)$ units of bandwidth of the link for the duration of the call. The goal of the link provider is to exercise call admission control in a way that maximizes the long term revenue.

Using uniformization, the problem is easily transformed into a discrete-time Markov decision process. The state can be taken to be of the form $i=(s(1), \ldots, s(M), \omega)$, where $s(m)$ denotes the number of active calls of type $m$, and $\omega$ indicates the type of event that triggers the next transition (a departure or arrival of a call, together with the type of the call). If $\omega$ indicates an arrival of a call of class $m$ and if there is enough free bandwidth to accommodate it, there are two available decisions, namely, $u_{a}$ (accept) or $u_{r}$ (reject).

We consider randomized policies of the following form. If there is an arrival of a call of class $m$, we accept it with probability

$$
\mu_{u_{a}}(i, \theta)=\frac{1}{1+\exp (s \cdot b-\theta(m))} .
$$

Here, $s \cdot b=\sum_{m} s(m) b(m)$ is the currently occupied bandwidth and $\theta(m)$, the $m$ th component of $\theta$, is a policy parameter. Note that

$$
\mu_{u_{a}}(i, \theta) \geq 0.5, \quad \text { if and only if } \quad s \cdot b \leq \theta(m)
$$

Thus, $\theta(m)$ can be interpreted as a "fuzzy" threshold on system occupancy, which determines whether type $m$ calls are to be admitted or rejected.

In our experiments, we consider a link with a total bandwidth of 10 units, and three different call types. The detailed parameters are given in Table I and correspond to a moderately-to-heavily loaded system. (If all calls were accepted, the link utilization would be $77.5 \%$ ). The number of link configurations (i.e., possible choices of $s$ that do not violate the link capacity constraint) turns out to be 286 .

Any state $(s, \omega)$ in which $s=(0, \ldots, 0)$ and $\omega$ corresponds to an arrival of a new call, is recurrent under any policy, and can therefore play the role of $i^{*}$.

\section{B. Results}

1) Optimal Policy: Since the state space is relatively small, an optimal policy can be obtained using standard dynamic programming methods [1]. The optimal average reward is equal to
TABLE I

CALL TYPES

\begin{tabular}{c|ccc}
\hline CALL TYPE $m$ & 1 & 2 & 3 \\
\hline & & & \\
$b(m)$ & 1 & 1 & 1 \\
$\alpha(m)$ & 1.8 & 1.6 & 1.4 \\
$1 / \beta(m)$ & $1 / 0.6$ & $1 / 0.5$ & $1 / 0.4$ \\
$c(m)$ & 1 & 2 & 4 \\
\hline
\end{tabular}
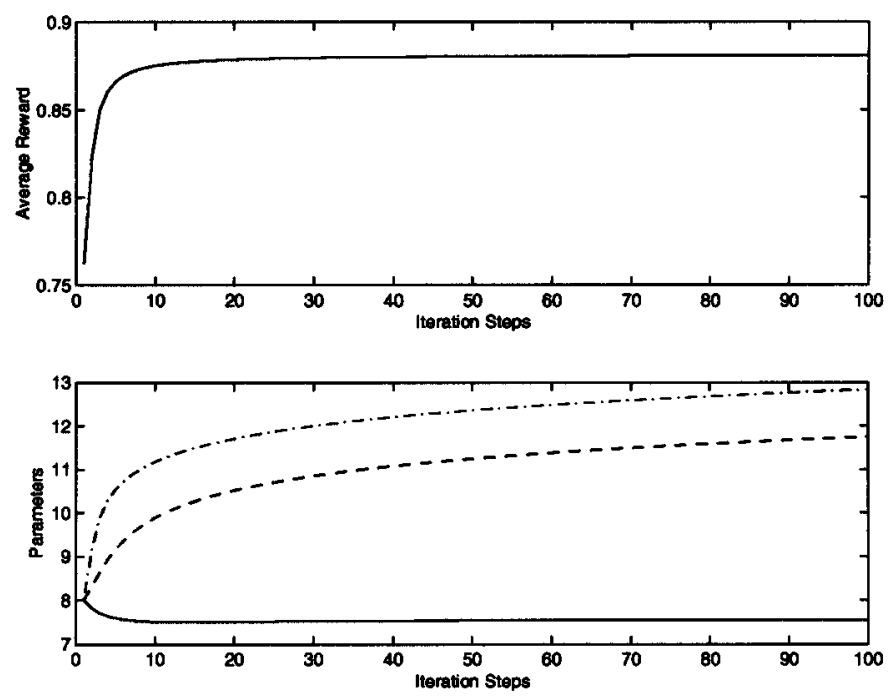

Fig. 1. Parameter vectors and average rewards (computed exactly) of the corresponding admission control policies, obtained by the idealized gradient algorithm. The solid, dashed, and dash-dot line correspond to the threshold values $\theta_{1}, \theta_{2}$, and $\theta_{3}$, associated with service types 1,2 , and 3 , respectively.

0.8868. (Of course, the optimal average reward within the restricted class of randomized policies that we have introduced earlier will have to be less than that.) Under an optimal policy, customers of type 2 and 3 are accepted whenever there is available bandwidth. Customers of type 1 are accepted only if the currently used bandwidth does not exceed 7 .

2) Idealized Gradient Algorithm: For such a small example, we can numerically calculate $\nabla \lambda(\theta)$, for any given $\theta$, which allows us to implement the idealized algorithm

$$
\theta_{k+1}=\theta_{k}+\gamma_{k} \nabla \lambda\left(\theta_{k}\right)
$$

of Section II-B. The evolution of this algorithm, starting with $\theta_{0}=(8,8,8)$, is shown in Fig. 1 . After 100 iterations, we have $\theta_{100}=(7.5459,11.7511,12.8339)$, and the corresponding average reward is equal to 0.8808 , which is very close to optimal. The probabilities of accepting a new call are given in Fig. 2 .

3) Simulation-Based Algorithm that Updates at Every Time Step: We implemented a streamlined version of the algorithm given Section IV, where we reset the vector $z_{k}$ not only at visits to the recurrent state $i^{*}$, but at visits to all states $i=(s, \omega)$ for which $s=(0, \ldots, 0)$. A justification of this modification, which does not change the mean direction of the update or the convergence properties, is given in [23]. In this version, the vector $z_{k}$ gets updated roughly every 10000 time steps. We started with the same initial parameter $\theta=(8,8,8)$, and the initial estimate of the average reward $\tilde{\lambda}_{0}$ was set to 0.78 . The scaling factor in the update equation was chosen to be $\eta=0.1$. 

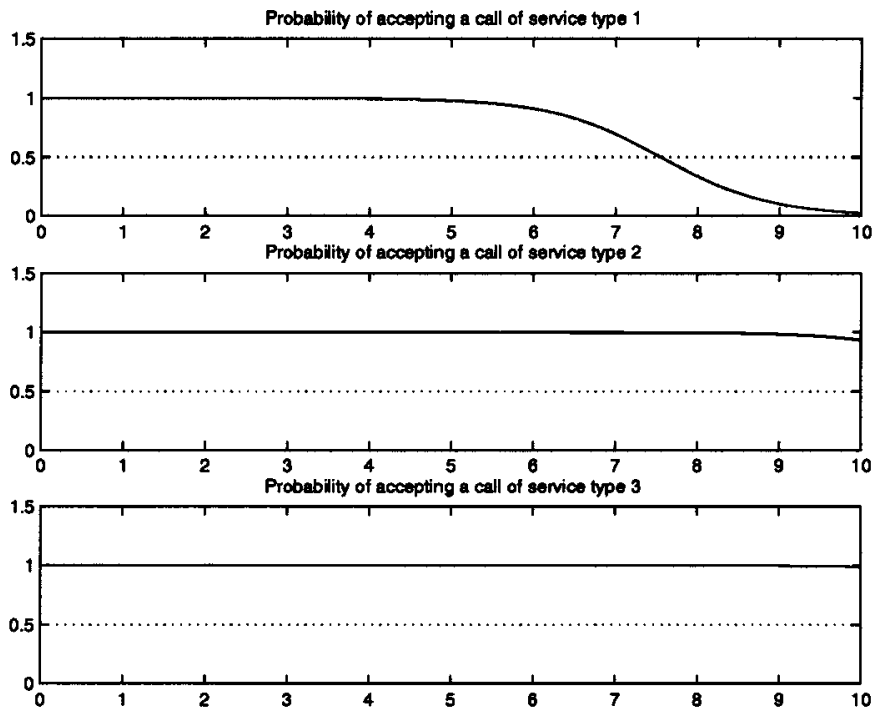

Fig. 2. Probabilities of accepting a new call, as a function of the already occupied bandwidth, under the control policy associated with the parameter vector $(7.5459,11.7511,12.8339)$ obtained by the idealized gradient algorithm.
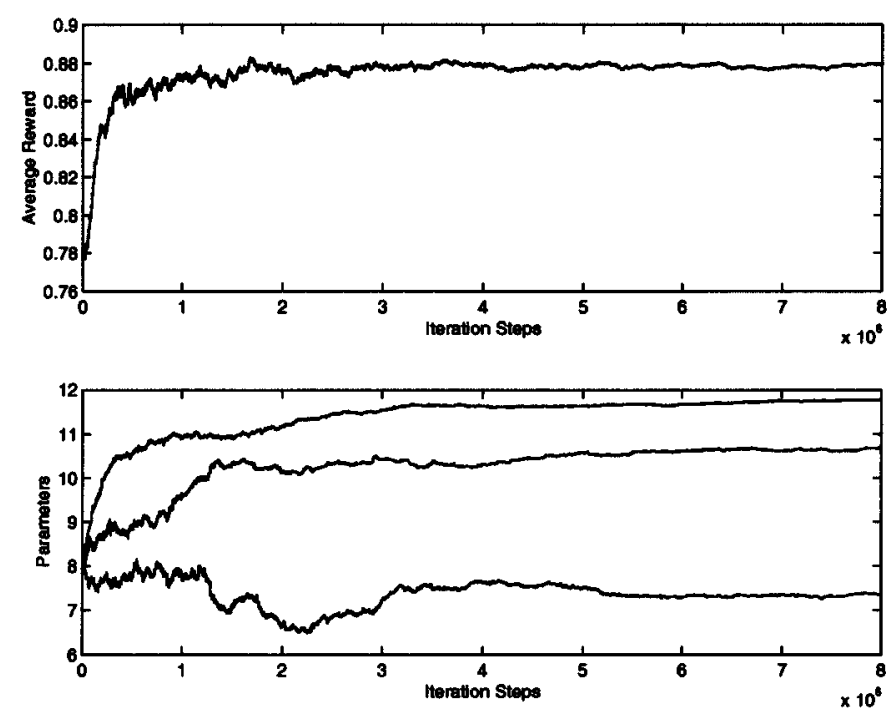

Fig. 3. Parameter vectors, and estimates of the average reward, obtained by the simulation-based algorithm. The scaling factor for the iteration steps is $10^{6}$.

The corresponding trajectories of the parameter vectors and average reward are given in Fig. 3. We have the following observations.

1) The algorithm makes rapid progress in the beginning, improving the average reward from 0.78 to 0.87 within the first $1 \cdot 10^{6}$ iteration steps.

2) After $1 \cdot 10^{6}$ iterations, the algorithm makes only slow progress obtaining after $8 \cdot 10^{6}$ iterations the parameter vector

$$
\theta_{8 \cdot 10^{6}}=(7.3540,10.6850,11.7713)
$$

which corresponds to an admission control policy with an average reward of 0.8789 . This average reward still slightly below the average reward of 0.8808 obtained by the idealized gradient algorithm.
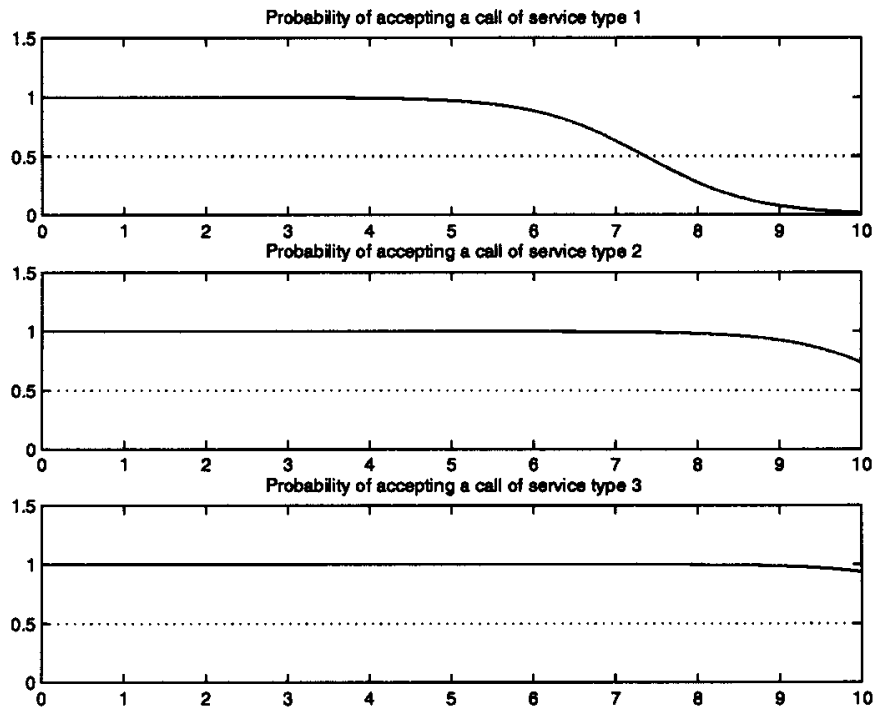

Fig. 4. Probabilities of accepting a new call, given as a function of the used bandwidth on the link, under the control policy associated with the parameter vector $(7.3540,10.6850,11.7713)$ obtained by the simulation-based algorithm.
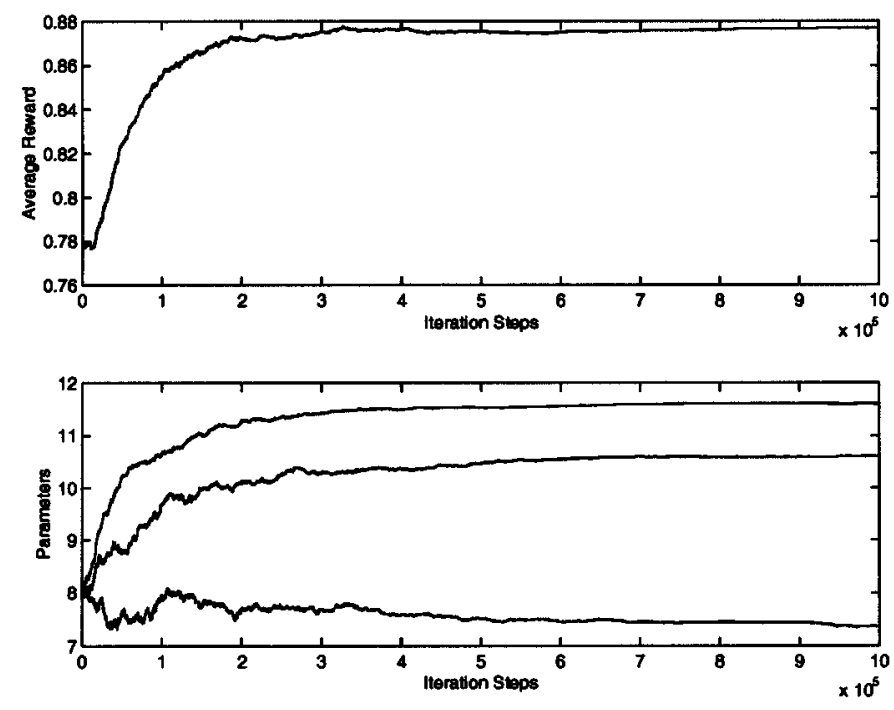

Fig. 5. Parameter vectors, and estimates of the average reward, obtained by modified simulation-based algorithm using a discount factor $\alpha=0.99$. The scaling factor for the iteration steps is $10^{5}$.

3) The fluctuations in the estimate of the average reward remain small and the performance of the control policies never deteriorates.

This behavior is not unlike the idealized algorithm (see Fig. 1), where the average reward improves rapidly in the beginning, but only slowly in the later iterations.

The probabilities of accepting a new call under the control policy obtained with the simulation-based algorithm are given in Fig. 4.

Modified Simulation-Based Algorithm: We finally consider the modified algorithm of Section $\mathrm{V}$, using a forgetting factor of $\alpha=0.99$. [Again, we reset the vector $z_{k}$ at visits to all states $i=(s, \omega)$ for which $s=(0, \ldots, 0)$.] As expected, it makes much faster progress; see Fig. 5.

After $10^{6}$ iterations, we obtain a parameter vector of $\theta=$ $(7.3553,10.6034,11.6073)$ and an average reward of 0.8785 , 
which is essentially the same as for the unmodified algorithm after $8 \cdot 10^{6}$ iterations. Thus, the use of a forgetting factor speeds up convergence by an order of magnitude, while introducing a negligible bias.

\section{CONCLUSION}

We have presented a simulation-based method for optimizing a Markov reward process whose transition probabilities depend on a parameter vector $\theta$, or a Markov decision process in which we restrict to a parametric set of randomized policies. The method involves simulation of a single sample path. Updates can be carried out either when the recurrent state $i^{*}$ is visited, or at every time step. In either case, the original method is expected to work well mostly when the regenerative cycles are not too long. We have also proposed a modified, possibly more practical method, and have provided some encouraging numerical results.

Regarding further research, there is a need for more computational experiments in order to delineate the class of practical problems for which this methodology is useful. In particular, further analysis and experimentation is needed for the modified on-line algorithm of Section V. In addition, the possibility of combining such methods with "learning" (function approximation) of the differential reward function needs to be explored. On the technical side, it may be possible to extend the results to the case of an infinite state space, and to relate the speed of convergence to the mixing time of the underlying Markov chains.

\section{APPENDIX I}

\section{PROOF OF PROPOSITION 3}

In this appendix, we prove convergence of the algorithm

$$
\begin{aligned}
& \theta_{m+1}=\theta_{m}+\gamma_{m} F_{m}\left(\theta_{m}, \tilde{\lambda}_{m}\right) \\
& \tilde{\lambda}_{m+1}=\tilde{\lambda}_{m}+\eta \gamma_{m} \sum_{n=t_{m}}^{t_{m+1}-1}\left(g_{i_{n}}\left(\theta_{m}\right)-\tilde{\lambda}_{m}\right)
\end{aligned}
$$

where

$$
\begin{aligned}
& F_{m}\left(\theta_{m}, \tilde{\lambda}_{m}\right) \\
& \quad=\sum_{n=t_{m}}^{t_{m+1}-1}\left(\tilde{v}_{i_{n}}\left(\theta_{m}, \tilde{\lambda}_{m}\right) L_{i_{n-1} i_{n}}\left(\theta_{m}\right)+\nabla g_{i_{n}}\left(\theta_{m}\right)\right) \\
& \tilde{v}_{i_{n}}(\theta, \tilde{\lambda}) \\
& \quad=\sum_{k=n}^{t_{m+1}-1}\left(g_{i_{k}}(\theta)-\tilde{\lambda}\right), \quad n=t_{m}+1, \cdots, t_{m+1}-1
\end{aligned}
$$

and

$$
\tilde{v}_{i_{m}}(\theta, \tilde{\lambda})=0
$$

For notational convenience, we define the augmented parameter vector $r_{m}=\left(\theta_{m}, \tilde{\lambda}_{m}\right)$, and write the update equations in the form

$$
r_{m+1}=r_{m}+\gamma_{m} H_{m}\left(r_{m}\right)
$$

where

$$
H_{m}\left(r_{m}\right)=\left[\begin{array}{c}
F_{m}\left(\theta_{m}, \tilde{\lambda}_{m}\right) \\
\eta \sum_{n=t_{m}}^{t_{m+1}-1}\left(g_{i_{n}}\left(\theta_{m}\right)-\tilde{\lambda}_{m}\right)
\end{array}\right]
$$

Let

$$
\mathcal{F}_{m}=\left\{\theta_{0}, \tilde{\lambda}_{0}, i_{0}, i_{1}, \ldots, i_{t_{m}}\right\}
$$

stand for the history of the algorithm up to and including time $t_{m}$. Using Proposition 2 and (17), we have

$$
E\left[H_{m}\left(r_{m}\right) \mid \mathcal{F}_{m}\right]=h\left(r_{m}\right)
$$

where

$$
h(r)=\left[\begin{array}{c}
E_{\theta}[T] \nabla \lambda(\theta)+G(\theta)(\lambda(\theta)-\tilde{\lambda}) \\
\eta E_{\theta}[T](\lambda(\theta)-\tilde{\lambda})
\end{array}\right] .
$$

We then rewrite the algorithm in the form

$$
r_{m+1}=r_{m}+\gamma_{m} h\left(r_{m}\right)+\varepsilon_{m}
$$

where

$$
\varepsilon_{m}=\gamma_{m}\left(H_{m}\left(r_{m}\right)-h\left(r_{m}\right)\right)
$$

and note that

$$
E\left[\varepsilon_{m} \mid \mathcal{F}_{m}\right]=0 .
$$

The proof rests on the fact that $\varepsilon_{m}$ is "small," in a sense to be made precise, which will then allow us to mimic the heuristic argument of Section III-C.

\section{A. Preliminaries}

In this subsection, we establish a few useful bounds and characterize the behavior of $\varepsilon_{m}$.

\section{Lemma 2:}

1) There exist constants $C$ and $\rho<1$ such that

$$
P_{\theta}(T=k) \leq C \rho^{k}, \quad \forall k, \theta
$$

where the subscript $\theta$ indicates that we are considering the distribution of the length of the regeneration cycle $T_{m}=$ $t_{m+1}-t_{m}$ under a particular choice of $\theta$. In particular, $E_{\theta}[T]$ and $E_{\theta}\left[T^{2}\right]$ are bounded functions of $\theta$.

2) The function $G(\theta)$ is well defined and bounded.

3) The sequence $\tilde{\lambda}_{m}$ is bounded, with probability 1 .

4) The sequence $h\left(r_{m}\right)$ is bounded, with probability 1 .

Proof:

1) For any transition probability matrix $P \in \overline{\mathcal{P}}$, and because of Assumption 1, the probability of reaching $i^{*}$ in $N$ steps is bounded below by some positive $\epsilon(P)$, for every initial state. Furthermore, $\epsilon(P)$ can be taken to be a continuous function of $P$. Using the compactness of $\overline{\mathcal{P}}$, we have $\epsilon^{*}=\min _{P \in \overline{\mathcal{P}}} \epsilon(P)>0$, and the result follows with $\rho=\left(\epsilon^{*}\right)^{1 / N}$. 
2) Note that

$E_{\theta}\left[\sum_{n=t_{m}+1}^{t_{m+1}-1}\left\|\left(t_{m+1}-n\right) L_{i_{n-1} i_{n}}(\theta)\right\|\right] \leq C E_{\theta}\left[T^{2}\right]$

where $C$ is a bound on $\left\|L_{i j}(\theta)\right\|$ (cf. Assumption 3). The right-hand side is bounded by the result of part a). It follows that the expectation defining $G(\theta)$ exists and is a bounded function of $\theta$.

3) Using Assumption 4 and part a) of this lemma, we obtain

$$
E\left[\sum_{m=1}^{\infty} \gamma_{m}^{2}\left(t_{m+1}-t_{m}\right)^{2}\right]<\infty
$$

which implies that $\gamma_{m}\left(t_{m+1}-t_{m}\right)$ converges to zero, with probability 1 . Note that

$\tilde{\lambda}_{m+1} \leq\left(1-\gamma_{m}\left(t_{m+1}-t_{m}\right)\right) \tilde{\lambda}_{m}+\gamma_{m}\left(t_{m+1}-t_{m}\right) C$

where $C$ is an upper bound on $g_{i}(\theta)$. For large enough $m$, we have $\gamma_{m}\left(t_{m+1}-t_{m}\right) \leq 1$, and $\tilde{\lambda}_{m+1} \leq \max \left\{\tilde{\lambda}_{m}, C\right\}$, from which it follows that the sequence $\tilde{\lambda}_{m}$ is bounded above. By a similar argument, the sequence $\tilde{\lambda}_{m}$ is also bounded below.

4) Consider the formula that defines $h(r)$. Parts a) and b) show that $E_{\theta_{m}}[T]$ and $G\left(\theta_{m}\right)$ are bounded. Also, $\lambda\left(\theta_{m}\right)$ is bounded since the $g_{i}(\theta)$ are bounded (Assumption 2). Furthermore, $\nabla \lambda\left(\theta_{m}\right)$ is bounded, by Lemma 1. Using also part c) of this lemma, the result follows.

Lemma 3: There exists a constant $C$ (which is random but finite with probability 1) such that

$$
E\left[\left\|\varepsilon_{m}\right\|^{2} \mid \mathcal{F}_{m}\right] \leq C \gamma_{m}^{2} \quad \forall m
$$

and the series $\sum_{m} \varepsilon_{m}$ converges with probability 1 .

Proof: Recall that $g_{i_{m}}\left(\theta_{m}\right)$ and $\tilde{\lambda}_{m}$ are bounded with probability 1 [Assumption 2 and Lemma 2c)]. Thus, for $n=t_{m}, \ldots, t_{m+1}-1$, we have $\left|\tilde{v}_{i_{n}}(\theta, \tilde{\lambda})\right| \leq C\left(t_{m+1}-t_{m}\right)$, for some constant $C$. Using this bound in the definition of $F_{m}\left(\theta_{m}, \tilde{\lambda}_{m}\right)$, we see that for almost all sample paths, we have

$$
\left\|F_{m}\left(\theta_{m}, \tilde{\lambda}_{m}\right)\right\| \leq C\left(t_{m+1}-t_{m}\right)^{2}
$$

for some new constant $C$. Using Lemma 2a), the conditional variance of $F_{m}\left(\theta_{m}, \tilde{\lambda}_{m}\right)$, given $\mathcal{F}_{m}$, is bounded. Similar arguments also apply to the last component of $H_{m}\left(r_{m}\right)$. Since $\varepsilon_{m}=\gamma_{m}\left(H_{m}\left(r_{m}\right)-E\left[H_{m}\left(r_{m}\right) \mid \mathcal{F}_{m}\right]\right)$, the first statement follows.

Fix a positive integer $c$ and consider the sequence

$$
w_{n}^{c}=\sum_{m=1}^{\min \{M(c), n\}} \varepsilon_{m}
$$

where $M(c)$ is the first time $m$ such that $E\left[\left\|\varepsilon_{m}\right\|^{2} \mid \mathcal{F}_{m}\right]>c \gamma_{m}^{2}$. The sequence $w_{n}^{c}$ is a martingale with bounded second moment, and therefore converges with probability 1 . This is true for every positive integer $c$. For (almost) every sample path, there exists some $c$ such that $M(c)=\infty$. After discarding a countable union of sets of measure zero (for each $c$, the set of sample paths for which $w_{n}^{c}$ does not converge), it follows that for (almost) every sample path, $\sum_{m} \varepsilon_{m}$ converges.
We observe the following consequences of Lemma 3. First, $\varepsilon_{m}$ converges to zero with probability 1 . Since $\gamma_{m}$ also converges to zero and the sequence $h\left(r_{m}\right)$ is bounded, we conclude that

$$
\lim _{m \rightarrow \infty}\left(\theta_{m+1}-\theta_{m}\right)=0, \quad \lim _{m \rightarrow \infty}\left(\lambda\left(\theta_{m+1}\right)-\lambda\left(\theta_{m}\right)\right)=0,
$$

and

$\lim _{m \rightarrow \infty}\left(\tilde{\lambda}_{m+1}-\tilde{\lambda}_{m}\right)=0$

with probability 1 .

\section{B. Convergence of $\tilde{\lambda}_{m}$ and $\lambda\left(\theta_{m}\right)$}

In this subsection, we prove that $\tilde{\lambda}_{m}$ and $\lambda\left(\theta_{m}\right)$ converge to a common limit. The flow of the proof is similar to the heuristic argument of III-C

We will be using a few different Lyapunov functions to analyze the behavior of the algorithm in different "regions." The lemma below involves a generic Lyapunov function $\phi$ and characterizes the changes in $\phi(r)$ caused by the updates

$$
r_{m+1}=r_{m}+\gamma_{m} h\left(r_{m}\right)+\varepsilon_{m} .
$$

Let $\mathcal{D}_{c}=\left\{(\theta, \tilde{\lambda}) \in \Re^{K+1}|| \tilde{\lambda} \mid \leq c\right\}$. We are interested in Lyapunov functions $\phi$ that are twice differentiable and for which $\phi, \nabla \phi$, and $\nabla^{2} \phi$ are bounded on $\mathcal{D}_{c}$ for every $c$. Let $\Phi$ be the set of all such Lyapunov functions. For any $\phi \in \Phi$, we define

$$
\varepsilon_{m}(\phi)=\phi\left(r_{m+1}\right)-\phi\left(r_{m}\right)-\gamma_{m} \nabla \phi\left(r_{m}\right) \cdot h\left(r_{m}\right)
$$

where for any two vectors $a, b$, we use $a \cdot b$ to denote their inner product.

Lemma 4: If $\phi \in \Phi$, then the series $\sum_{m} \varepsilon_{m}(\phi)$ converges with probability 1 .

Proof: Consider a sample path of the random sequence $\left\{r_{m}\right\}$. Using part 3) of Lemma 2, and after discarding a set of zero probability, there exists some $c$ such that $r_{m} \in \mathcal{D}_{c}$ for all $m$. We use the Taylor expansion of $\phi(r)$ at $r_{m}$, and obtain

$$
\begin{aligned}
\varepsilon_{m}(\phi)= & \phi\left(r_{m+1}\right)-\phi\left(r_{m}\right)-\gamma_{m} \nabla \phi\left(r_{m}\right) \cdot h\left(r_{m}\right) \\
\leq & \nabla \phi\left(r_{m}\right) \cdot\left(r_{m+1}-r_{m}\right)+M\left\|r_{m+1}-r_{m}\right\|^{2} \\
& -\gamma_{m} \nabla \phi\left(r_{m}\right) \cdot h\left(r_{m}\right) \\
= & \nabla \phi\left(r_{m}\right) \cdot \varepsilon_{m}+M\left\|r_{m+1}-r_{m}\right\|^{2}
\end{aligned}
$$

where $M$ is a constant related to the bound on the second derivatives of $\phi(\cdot)$ on the set $\mathcal{D}_{c}$. A symmetric argument also yields

$$
\nabla \phi\left(r_{m}\right) \cdot \varepsilon_{m}-M\left\|r_{m+1}-r_{m}\right\|^{2} \leq \varepsilon_{m}(\phi) .
$$

Using the boundedness of $\nabla \phi$ on the set $\mathcal{D}_{c}$, the same martingale argument as in the proof of Lemma 3 shows that the series $\sum_{m} \nabla \phi\left(r_{m}\right) \cdot \varepsilon_{m}$ converges with probability 1 . Note that $\left\|r_{m+1}-r_{m}\right\|=\left\|\gamma_{m} h\left(r_{m}\right)+\varepsilon_{m}\right\|$, which yields

$$
\left\|r_{m+1}-r_{m}\right\|^{2} \leq 2 \gamma_{m}^{2}\left\|h\left(r_{m}\right)\right\|^{2}+2\left\|\varepsilon_{m}\right\|^{2} \text {. }
$$

The sequence $h\left(r_{m}\right)$ is bounded (Lemma 2) and $\gamma_{m}^{2}$ is summable (Assumption 4). Furthermore, it is an easy consequence of Lemma 3 that $\varepsilon_{m}$ is also square summable. We conclude that $\left\|r_{m+1}-r_{m}\right\|$ is square summable, and the result follows.

From now on, we will concentrate on a single sample path for which the sequences $\varepsilon_{m}$ and $\varepsilon_{m}(\phi)$ (for the Lyapunov func- 
tions to be considered) are summable. Accordingly, we will be omitting the "with probability 1" qualification.

The next lemma shows that if the error $\tilde{\lambda}_{m}-\lambda\left(\theta_{m}\right)$ in estimating the average reward is positive but small, then it tends to decrease. The proof uses $\tilde{\lambda}-\lambda(\theta)$ as a Lyapunov function.

Lemma 5: Let $L$ be such that $\|G(\theta)\| \leq L$ for all $\theta$, and let

$$
\phi(r)=\phi(\theta, \tilde{\lambda})=\tilde{\lambda}-\lambda(\theta) .
$$

We have $\phi \in \Phi$. Furthermore, if $0 \leq \tilde{\lambda}-\lambda(\theta) \leq \eta / L^{2}$, then

$$
\nabla \phi(r) \cdot h(r) \leq 0 .
$$

Proof: The fact that $\phi \in \Phi$ is a consequence of Lemma 1. We now have

$$
\begin{aligned}
\nabla \phi(r) \cdot h(r)= & -\eta(\tilde{\lambda}-\lambda(\theta)) E_{\theta}[T] \\
& -\|\nabla \lambda(\theta)\|^{2} E_{\theta}[T] \\
& +(\tilde{\lambda}-\lambda(\theta)) \nabla \lambda(\theta) \cdot G(\theta) .
\end{aligned}
$$

Using the inequality $|a \cdot b| \leq\|a\|^{2}+\|b\|^{2}$, to bound the last term, and the fact $E_{\theta}[T] \geq 1$, we obtain

$$
\nabla \phi(r) \cdot h(r) \leq-\eta(\tilde{\lambda}-\lambda(\theta))+L^{2}(\tilde{\lambda}-\lambda(\theta))^{2}
$$

which is nonpositive as long as $0 \leq \tilde{\lambda}-\lambda(\theta) \leq \eta / L^{2}$.

In the next two lemmas, we establish that if $\left|\tilde{\lambda}_{m}-\lambda\left(\theta_{m}\right)\right|$ remains small during a certain time interval, then $\tilde{\lambda}_{m}$ cannot decrease by much. We first introduce a Lyapunov function that captures the behavior of the algorithm when $\tilde{\lambda} \approx \lambda(\theta)$.

Lemma 6: As in Lemma 5, let $L$ be such that $\|G(\theta)\| \leq L$. Let also

$$
\phi(r)=\phi(\theta, \tilde{\lambda})=\lambda(\theta)-\left(L^{2} / \eta\right)(\lambda(\theta)-\tilde{\lambda})^{2} .
$$

We have $\phi \in \Phi$. Furthermore, if $|\lambda(\theta)-\tilde{\lambda}| \leq \eta / 4 L^{2}$, then

$$
\nabla \phi(r) \cdot h(r) \geq 0 .
$$

Proof: The fact that $\phi \in \Phi$ is a consequence of Lemma 1. We have

and

$$
\nabla_{\theta} \phi(\theta, \tilde{\lambda})=\left(1-\left(2 L^{2} / \eta\right)(\lambda(\theta)-\tilde{\lambda})\right) \nabla \lambda(\theta)
$$

$$
\nabla_{\tilde{\lambda}} \phi(\theta, \tilde{\lambda})=\left(2 L^{2} / \eta\right)(\lambda(\theta)-\tilde{\lambda}) .
$$

Therefore, assuming that $|\lambda(\theta)-\tilde{\lambda}| \leq \eta / 4 L^{2}$, and using the Schwartz inequality, we obtain

$$
\begin{aligned}
\nabla \phi(r) & \cdot h(r) \\
= & \left(1-\left(2 L^{2} / \eta\right)(\lambda(\theta)-\tilde{\lambda})\right) \\
& \times\left(\|\nabla \lambda(\theta)\|^{2} E_{\theta}[T]+(\lambda(\theta)-\tilde{\lambda}) G(\theta) \cdot \nabla \lambda(\theta)\right) \\
& +2 L^{2}(\lambda(\theta)-\tilde{\lambda})^{2} E_{\theta}[T] \\
\geq & \frac{1}{2}\|\nabla \lambda(\theta)\|^{2}-\frac{3}{2}|\lambda(\theta)-\tilde{\lambda}| L\|\nabla \lambda(\theta)\| \\
& +2 L^{2}(\lambda(\theta)-\tilde{\lambda})^{2} \geq 0 .
\end{aligned}
$$

Lemma 7: Consider the same function $\phi$ as in Lemma 6, and the same constant $L$. Let $\alpha$ be some positive scalar smaller than $\eta / 4 L^{2}$. Suppose that for some integers $n$ and $n^{\prime}$, with $n^{\prime}>n$, we have

and

$$
\left|\lambda\left(\theta_{n}\right)-\tilde{\lambda}_{n}\right| \leq \alpha, \quad\left|\lambda\left(\theta_{n^{\prime}}\right)-\tilde{\lambda}_{n^{\prime}}\right| \leq \alpha,
$$

$$
\left|\lambda\left(\theta_{m}\right)-\tilde{\lambda}_{m}\right| \leq \frac{\eta}{4 L^{2}}, \quad m=n+1, \ldots, n^{\prime}-1 .
$$

Then,

$$
\tilde{\lambda}_{n^{\prime}} \geq \tilde{\lambda}_{n}-2 \alpha\left(\left(L^{2} \alpha / \eta\right)+1\right)+\sum_{m=n}^{n^{\prime}-1} \varepsilon_{m}(\phi) .
$$

Proof: Using Lemma 6, we have

$$
\nabla \phi\left(r_{m}\right) \cdot h\left(r_{m}\right) \geq 0, \quad m=n, \ldots, n^{\prime}-1 .
$$

Therefore, for $m=n, \ldots, n^{\prime}-1$, we have

$$
\begin{aligned}
\phi\left(r_{m+1}\right) & =\phi\left(r_{m}\right)+\gamma_{m} \nabla \phi\left(r_{m}\right) \cdot h\left(r_{m}\right)+\varepsilon_{m}(\phi) \\
& \geq \phi\left(r_{m}\right)+\varepsilon_{m}(\phi),
\end{aligned}
$$

and

$$
\phi\left(r_{n^{\prime}}\right) \geq \phi\left(r_{n}\right)+\sum_{m=n}^{n^{\prime}-1} \varepsilon_{m}(\phi) .
$$

Note that $\left|\phi\left(r_{n}\right)-\tilde{\lambda}_{n}\right| \leq\left(L^{2} \alpha^{2} / \eta\right)+\alpha$, and $\left|\phi\left(r_{n^{\prime}}\right)-\tilde{\lambda}_{n^{\prime}}\right| \leq$ $\left(L^{2} \alpha^{2} / \eta\right)+\alpha$. Using these inequalities in (26), we obtain the desired result.

Lemma 8: We have $\lim \inf _{m \rightarrow \infty}\left|\lambda\left(\theta_{m}\right)-\tilde{\lambda}_{m}\right|=0$.

Proof: Suppose that the result is not true, and we will derive a contradiction. Since $\lambda\left(\theta_{m+1}\right)-\lambda\left(\theta_{m}\right)$ and $\tilde{\lambda}_{m+1}-\tilde{\lambda}_{m}$ converge to zero, there exists a scalar $\epsilon>0$ and an integer $n$, such that either $\lambda\left(\theta_{m}\right)-\tilde{\lambda}_{m}>\epsilon$, or $\lambda\left(\theta_{m}\right)-\tilde{\lambda}_{m}<-\epsilon$, for all $m>n$. Without loss of generality, let us consider the first possibility.

Recall that the update equation for $\tilde{\lambda}$ is of the form

$$
\tilde{\lambda}_{m+1}=\tilde{\lambda}_{m}+\eta \gamma_{m} E_{\theta_{m}}[T]\left(\lambda\left(\theta_{m}\right)-\tilde{\lambda}_{m}\right)+\delta_{m}
$$

where $\delta_{m}$ is the last component of the vector $\varepsilon_{m}$, which is summable by Lemma 3. Given that $\lambda\left(\theta_{m}\right)-\tilde{\lambda}_{m}$ stays above $\epsilon$, the sequence $\eta \gamma_{m}\left(\lambda\left(\theta_{m}\right)-\tilde{\lambda}_{m}\right)$ sums to infinity. As $\delta_{m}$ is summable, we conclude that $\tilde{\lambda}_{m}$ converges to infinity, which contradicts the fact that it is bounded.

The next lemma shows that the condition $\lambda\left(\theta_{m}\right) \geq \tilde{\lambda}_{m}$ is satisfied, in the limit.

Lemma 9: We have $\liminf _{m \rightarrow \infty}\left(\lambda\left(\theta_{m}\right)-\tilde{\lambda}_{m}\right) \geq 0$.

Proof: Suppose the contrary. Then, there exists some $\epsilon>$ 0 such that the inequality

$$
\tilde{\lambda}_{m}-\lambda\left(\theta_{m}\right)>\epsilon
$$

holds infinitely often. Let $\beta=\min \left\{\epsilon, \eta / L^{2}\right\}$, where $L$ is the constant of Lemma 5. Using Lemma 8, we conclude that $\tilde{\lambda}_{m}-$ $\lambda\left(\theta_{m}\right)$ crosses infinitely often from a value smaller than $\beta / 3$ 
to a value larger than $2 \beta / 3$. In particular, there exist infinitely many pairs $n, n^{\prime}$, with $n^{\prime}>n$, such that

and

$$
0<\tilde{\lambda}_{n}-\lambda\left(\theta_{n}\right)<\frac{1}{3} \beta, \quad \tilde{\lambda}_{n^{\prime}}-\lambda\left(\theta_{n^{\prime}}\right)>\frac{2}{3} \beta
$$

$$
\frac{1}{3} \beta \leq \tilde{\lambda}_{m}-\lambda\left(\theta_{m}\right) \leq \frac{2}{3} \beta, \quad m=n+1, \ldots, n^{\prime}-1 .
$$

We use the Lyapunov function

$$
\phi(r)=\phi(\theta, \tilde{\lambda})=\tilde{\lambda}-\lambda(\theta)
$$

and note that

$$
\phi\left(r_{n^{\prime}}\right) \geq \phi\left(r_{n}\right)+\frac{\beta}{3} .
$$

For $m=n, \ldots, n^{\prime}-1$, we have $0<\tilde{\lambda}-\lambda(\theta)<\beta \leq$ $\eta / L^{2}$. Lemma 5 applies and shows that $\nabla \phi\left(r_{m}\right) \cdot h\left(r_{m}\right) \leq 0$. Therefore,

$$
\begin{aligned}
\phi\left(r_{n^{\prime}}\right) & =\phi\left(r_{n}\right)+\sum_{m=n}^{n^{\prime}-1}\left(\gamma_{m} \nabla \phi\left(r_{m}\right) \cdot h\left(r_{m}\right)+\varepsilon_{m}(\phi)\right) \\
& \leq \phi\left(r_{n}\right)+\sum_{m=n}^{n^{\prime}-1} \varepsilon_{m}(\phi) .
\end{aligned}
$$

By Lemma $4, \sum_{m} \varepsilon_{m}(\phi)$ converges, which implies that $\sum_{m=n}^{n^{\prime}-1} \varepsilon_{m}(\phi)$ becomes arbitrarily small. This contradicts (27) and completes the proof.

We now continue with the central step in the proof, which consists of showing that $\lim _{m \rightarrow \infty}\left(\lambda\left(\theta_{m}\right)-\tilde{\lambda}_{m}\right)=0$. Using Lemma 9, it suffices to show that we cannot have $\limsup _{m \rightarrow \infty}\left(\lambda\left(\theta_{m}\right)-\tilde{\lambda}_{m}\right)>0$. The main idea is the following. Whenever $\lambda\left(\theta_{m}\right)$ becomes significantly larger than $\tilde{\lambda}_{m}$, then $\tilde{\lambda}_{m}$ is bound to increase significantly. On the other hand, by Lemma 7, whenever $\lambda\left(\theta_{m}\right)$ is approximately equal to $\tilde{\lambda}_{m}$, then $\tilde{\lambda}_{m}$ cannot decrease by much. Since $\tilde{\lambda}_{m}$ is bounded, this will imply that $\lambda\left(\theta_{m}\right)$ can become significantly larger than $\tilde{\lambda}_{m}$ only a finite number of times.

Lemma 10: We have $\lim _{m \rightarrow \infty}\left(\lambda\left(\theta_{m}\right)-\tilde{\lambda}_{m}\right)=0$.

Proof: We will assume the contrary and derive a contradiction. By Lemma 9, we have

$$
\liminf _{m \rightarrow \infty}\left(\lambda\left(\theta_{m}\right)-\tilde{\lambda}_{m}\right) \geq 0 .
$$

So if the desired result is not true, we must have $\limsup _{m \rightarrow \infty}\left(\lambda\left(\theta_{m}\right)-\tilde{\lambda}_{m}\right)>0$, which we will assume to be the case. In particular, there is some $A>0$ such that $\lambda\left(\theta_{m}\right)-\tilde{\lambda}_{m}>A$, infinitely often. Without loss of generality, we assume that $A \leq \eta / 4 L^{2}$, where $L$ is the constant of Lemmas 5 and 6 . Let $\alpha>0$ be some small constant (with $\alpha<A / 2$ ), to be specified later. Using Lemma 9, we have $\lambda\left(\theta_{m}\right)-\tilde{\lambda}_{m}>-\alpha$ for all large enough $m$. In addition, by Lemma 8 , the condition $\left|\lambda\left(\theta_{m}\right)-\tilde{\lambda}_{m}\right| \leq \alpha$ holds infinitely often. Thus, the algorithm can be broken down into a sequence of cycles, where in the beginning and at the end of each cycle we have $\left|\lambda\left(\theta_{m}\right)-\tilde{\lambda}_{m}\right| \leq \alpha$, while the condition $\lambda\left(\theta_{m}\right)-\tilde{\lambda}_{m}>A$ holds at some intermediate time in the cycle.
We describe the stages of such a cycle more precisely. A typical cycle starts at some time $N$ with $\left|\lambda\left(\theta_{N}\right)-\tilde{\lambda}_{N}\right| \leq \alpha$. Let $n^{\prime \prime}$ be the first time after time $N$ that $\lambda\left(\theta_{n^{\prime \prime}}\right)-\tilde{\lambda}_{n^{\prime \prime}}>A$. Let $n^{\prime}$ be the last time before $n^{\prime \prime}$ such that $\lambda\left(\theta_{n^{\prime}}\right)-\tilde{\lambda}_{n^{\prime}}<A / 2$. Let also $n$ be the last time before $n^{\prime}$ such that $\lambda\left(\theta_{n}\right)-\tilde{\lambda}_{n}<\alpha$. Finally, let $n^{\prime \prime \prime}$ be the first time after $n^{\prime \prime}$ such that $\left|\lambda\left(\theta_{n^{\prime \prime \prime}}\right)-\tilde{\lambda}_{n^{\prime \prime \prime}}\right|<\alpha$. The time $n^{\prime \prime \prime}$ is the end of the cycle and marks the beginning of a new cycle.

Recall that the changes in $\theta_{m}$ and $\tilde{\lambda}_{m}$ converge to zero. For this reason, by taking $N$ to be large enough, we can assume that $\lambda\left(\theta_{n}\right)-\tilde{\lambda}_{n} \geq 0$. To summarize our construction, we have $N<n<n^{\prime}<n^{\prime \prime}<n^{\prime \prime \prime}$, and

$$
\begin{gathered}
\left|\lambda\left(\theta_{N}\right)-\tilde{\lambda}_{N}\right|<\alpha, \quad 0 \leq \lambda\left(\theta_{n}\right)-\tilde{\lambda}_{n}<\alpha, \\
\left|\lambda\left(\theta_{m}\right)-\tilde{\lambda}_{m}\right| \leq A, \quad m=N, \ldots, n^{\prime \prime}-1, \\
\lambda\left(\theta_{n^{\prime}}\right)-\tilde{\lambda}_{n^{\prime}}<\frac{A}{2}, \quad \lambda\left(\theta_{n^{\prime \prime}}\right)-\tilde{\lambda}_{n^{\prime \prime}}>A \\
\alpha \leq \lambda\left(\theta_{m}\right)-\tilde{\lambda}_{m} \leq A, \quad m=n+1, \ldots, n^{\prime \prime}-1 \\
\frac{A}{2} \leq \lambda\left(\theta_{m}\right)-\tilde{\lambda}_{m} \leq A, \quad m=n^{\prime}+1, \ldots, n^{\prime \prime}-1 \\
\alpha \leq \lambda\left(\theta_{m}\right)-\tilde{\lambda}_{m}, \quad m=n^{\prime \prime}, \ldots, n^{\prime \prime \prime}-1 .
\end{gathered}
$$

Our argument will use the Lyapunov functions

$$
\phi(r)=\phi(\theta, \tilde{\lambda})=\lambda(\theta)-\left(L^{2} / \eta\right)(\lambda(\theta)-\tilde{\lambda})^{2}
$$

where $L$ is as in Lemma 5 and 6, and

$$
\psi(r)=\psi(\theta, \tilde{\lambda})=\tilde{\lambda}-\lambda(\theta) .
$$

We have

$$
\varepsilon_{m}(\phi)=\phi\left(r_{m+1}\right)-\phi\left(r_{m}\right)-\gamma_{m} \nabla \phi\left(r_{m}\right) \cdot h\left(r_{m}\right)
$$

and we define $\varepsilon_{m}(\psi)$ by a similar formula. By Lemma 4, the series $\sum_{m} \varepsilon_{m}(\phi)$ and $\sum_{m} \varepsilon_{m}(\psi)$ converge. Also, let

$$
\delta_{m}=\tilde{\lambda}_{m+1}-\tilde{\lambda}_{m}-\eta \gamma_{m} E_{\theta_{m}}[T]\left(\lambda\left(\theta_{m}\right)-\tilde{\lambda}_{m}\right) .
$$

We observe that $\delta_{m}$ is the last component of $\varepsilon_{m}$ and therefore, the series $\sum_{m} \delta_{m}$ converges and $\lim _{m \rightarrow \infty} \delta_{m}=0$. Finally, let $C$ be a constant such that $\left|\nabla \psi\left(r_{m}\right) \cdot h\left(r_{m}\right)\right| \leq C$, for all $m$, which exists because $\psi \in \Phi$ and because the sequences $h\left(r_{m}\right)$ and $\tilde{\lambda}_{m}$ are bounded.

Using the above observations, we see that if the beginning time $N$ of a cycle is chosen large enough, then for any $k, k^{\prime}$ such that $N \leq k \leq k^{\prime}$, we have

$$
\begin{aligned}
& \gamma_{k} C \leq \frac{A}{32}, \quad\left|\sum_{m=k}^{k^{\prime}} \varepsilon_{m}(\phi)\right| \leq \frac{A^{2}}{96 C} \\
& \left|\sum_{m=k}^{k^{\prime}} \varepsilon_{m}(\psi)\right| \leq \frac{A}{32}, \quad\left|\sum_{m=k}^{k^{\prime}} \delta_{m}\right| \leq \eta \frac{A^{2}}{8 C} .
\end{aligned}
$$


Finally, we assume that $\alpha$ has been chosen small enough so that

$$
2\left(\alpha+\left(L^{2} \alpha^{2} / \eta\right)\right) \leq \eta \frac{A^{2}}{96 C}
$$

Using the fact that $\lambda\left(\theta_{n^{\prime}+1}\right)-\tilde{\lambda}_{n^{\prime}+1} \geq A / 2$, we have

$$
\begin{aligned}
\lambda\left(\theta_{n^{\prime}}\right)-\tilde{\lambda}_{n^{\prime}}= & \lambda\left(\theta_{n^{\prime}+1}\right)-\tilde{\lambda}_{n^{\prime}+1}+\gamma_{n^{\prime}} \nabla \psi\left(r_{n^{\prime}}\right) \\
& \cdot h\left(r_{n^{\prime}}\right)+\varepsilon_{n^{\prime}}(\psi) \\
\geq & \frac{A}{2}-\frac{A}{16} .
\end{aligned}
$$

Furthermore, we have

$$
\begin{aligned}
\frac{A}{2} & \leq\left(\left(\lambda\left(\theta_{n^{\prime \prime}}\right)-\tilde{\lambda}_{n^{\prime \prime}}\right)-\left(\lambda\left(\theta_{n^{\prime}}\right)-\tilde{\lambda}_{n^{\prime}}\right)\right) \\
& =-\psi\left(r_{n^{\prime \prime}}\right)+\psi\left(r_{n^{\prime}}\right) \\
& =-\sum_{m=n^{\prime}}^{n^{\prime \prime}-1} \gamma_{m} \nabla \psi\left(r_{m}\right) \cdot h\left(r_{m}\right)-\sum_{m=n^{\prime}}^{n^{\prime \prime}-1} \varepsilon_{m}(\psi) \\
& \leq \sum_{m=n^{\prime}}^{n^{\prime \prime}-1} \gamma_{m} C+\frac{A}{32}
\end{aligned}
$$

which implies that

$$
\sum_{m=n^{\prime}}^{n^{\prime \prime}-1} \gamma_{m} \geq \frac{A}{2 C}-\frac{A}{32 C}
$$

Then,

$$
\begin{aligned}
\tilde{\lambda}_{n^{\prime \prime \prime}} & =\tilde{\lambda}_{n}+\sum_{m=n}^{n^{\prime \prime \prime}-1} \eta \gamma_{m} E_{\theta_{m}}[T]\left(\lambda\left(\theta_{m}\right)-\tilde{\lambda}_{m}\right)+\sum_{m=n}^{n^{\prime \prime}-1} \delta_{m} \\
& \geq \tilde{\lambda}_{n}+\sum_{m=n^{\prime}}^{n^{\prime \prime}-1} \eta \gamma_{m}\left(\lambda\left(\theta_{m}\right)-\tilde{\lambda}_{m}\right)+\sum_{m=n}^{n^{\prime \prime \prime}-1} \delta_{m} \\
& \geq \tilde{\lambda}_{n}+\eta\left(\frac{A}{2 C}-\frac{A}{32 C}\right)\left(\frac{A}{2}-\frac{A}{16}\right)-\eta \frac{A^{2}}{8 C} \\
& \geq \tilde{\lambda}_{n}+\eta \frac{A^{2}}{24 C}
\end{aligned}
$$

We have shown so far that $\tilde{\lambda}_{m}$ has a substantial increase between time $n$ and $n^{\prime \prime \prime}$. We now show that $\tilde{\lambda}_{m}$ can only have a small decrease in the time between $N$ and $n$. Indeed, by Lemma 7, we have

$$
\tilde{\lambda}_{n} \geq \tilde{\lambda}_{N}-2\left(\alpha+L^{2} \alpha^{2}\right)+\sum_{m=N}^{n-1} \varepsilon_{m}(\phi) .
$$

By combining these two properties, we obtain

$$
\begin{aligned}
\tilde{\lambda}_{n^{\prime \prime \prime}} & \geq \tilde{\lambda}_{N}-2\left(\alpha+L^{2} \alpha^{2}\right)-\eta \frac{A^{2}}{96 C}+\eta \frac{A^{2}}{24 C} \\
& \geq \tilde{\lambda}_{N}+\eta \frac{A^{2}}{48 C} .
\end{aligned}
$$

We have shown that $\tilde{\lambda}_{m}$ increases by a positive amount during each cycle. Since $\tilde{\lambda}_{m}$ is bounded above, this proves that there can only be a finite number of cycles, and a contradiction has been obtained.

Lemma 11: The sequences $\tilde{\lambda}_{m}$ and $\lambda\left(\theta_{m}\right)$ converge.

Proof: Consider the function $\phi(r)=\lambda(\theta)-$ $\left(L^{2} / \eta\right)(\lambda(\theta)-\tilde{\lambda})^{2}$, and the same constant $L$ as in Lemma 6. Let $\alpha$ be a scalar such that $0<\alpha \leq \eta /\left(4 L^{2}\right)$. By the preceding lemma and by Lemma 4, there exists some $N$ such that if $N \leq n \leq n^{\prime}$, we have

and

$$
\left|\lambda\left(\theta_{n}\right)-\tilde{\lambda}_{n}\right| \leq \alpha
$$

$$
\left|\sum_{m=n}^{n^{\prime}-1} \varepsilon_{m}(\phi)\right| \leq \alpha
$$

Using Lemma 6,

$$
\begin{gathered}
\phi\left(\theta_{n^{\prime}}\right) \geq \phi\left(\theta_{n}\right)+\sum_{m=n}^{n^{\prime}-1} \varepsilon_{m}(\phi) \geq \phi\left(\theta_{n}\right)-\alpha, \\
N \leq n \leq n^{\prime}
\end{gathered}
$$

or

$$
\begin{aligned}
& \lambda\left(\theta_{n^{\prime}}\right)-\left(L^{2} / \eta\right)\left(\lambda\left(\theta_{n^{\prime}}\right)-\tilde{\lambda}_{n^{\prime}}\right)^{2} \\
& \quad \geq \lambda\left(\theta_{n}\right)-\left(L^{2} / \eta\right)\left(\lambda\left(\theta_{n}\right)-\tilde{\lambda}_{n}\right)^{2}-\alpha
\end{aligned}
$$

which implies

$$
\lambda\left(\theta_{n^{\prime}}\right) \geq \lambda\left(\theta_{n}\right)-\left(L^{2} \alpha^{2} / \eta\right)-\alpha, \quad N \leq n \leq n^{\prime}
$$

Therefore,

$$
\liminf _{n^{\prime} \rightarrow \infty} \lambda\left(\theta_{n^{\prime}}\right) \geq \lambda\left(\theta_{n}\right)-\left(L^{2} \alpha^{2} / \eta\right)-\alpha, \quad N \leq n
$$

and this implies that

$$
\liminf _{m \rightarrow \infty} \lambda\left(\theta_{m}\right) \geq \limsup _{m \rightarrow \infty} \lambda\left(\theta_{m}\right)-\left(L^{2} \alpha^{2} / \eta\right)-\alpha
$$

Since $\alpha$ can be chosen arbitrarily small, we have $\liminf _{m \rightarrow \infty} \lambda\left(\theta_{m}\right) \geq \limsup _{m \rightarrow \infty} \lambda\left(\theta_{m}\right)$, and since the sequence $\lambda\left(\theta_{m}\right)$ is bounded, we conclude that it converges. Using also Lemma 10 , it follows that $\tilde{\lambda}_{m}$ converges as well.

\section{Convergence of $\nabla \lambda\left(\theta_{m}\right)$}

In the preceding subsection, we have shown that $\lambda\left(\theta_{m}\right)$ and $\tilde{\lambda}_{m}$ converge to a common limit. It now remains to show that $\nabla \lambda\left(\theta_{m}\right)$ converges to zero.

Since $\lambda\left(\theta_{t_{m}}\right)-\tilde{\lambda}_{t_{m}}$ converges to zero, the algorithm is of the form

$$
\theta_{m+1}=\theta_{m}+\gamma_{m} E_{\theta_{m}}[T]\left(\nabla \lambda\left(\theta_{m}\right)+e_{m}\right)+\epsilon_{m}
$$

where $e_{m}$ converges to zero and $\epsilon_{m}$ is a summable sequence. This is a gradient method with errors, similar to the methods considered in [10] and [6]. However, [10] assumes the boundedness of the sequence of iterates, and the results of [6] do not 
include the term $e_{m}$. Thus, while the situation is very similar to that considered in these references, a separate proof is needed.

We will first show that $\liminf _{m \rightarrow \infty}\left\|\nabla \lambda\left(\theta_{m}\right)\right\|=0$. Suppose the contrary. Then, there exists some $\epsilon>0$ and some $N$ such that $\left\|\nabla \lambda\left(\theta_{m}\right)\right\|>\epsilon$ for all $m>N$. In addition, by taking $N$ large enough, we can also assume that $\left\|e_{m}\right\| \leq \epsilon / 2$. Then, it is easily checked that

$$
\nabla \lambda\left(\theta_{m}\right) \cdot\left(\nabla \lambda\left(\theta_{m}\right)+e_{m}\right) \geq \frac{\epsilon^{2}}{2} .
$$

Let $\phi(r)=\lambda(\theta)$. Note that $\phi \in \Phi$. We have

$$
\begin{aligned}
\lambda\left(\theta_{m+1}\right)= & \lambda\left(\theta_{m}\right)+\gamma_{m} E_{\theta_{m}}[T] \nabla \lambda\left(\theta_{m}\right) \\
& \cdot\left(\nabla \lambda\left(\theta_{m}\right)+e_{m}\right)+\varepsilon_{m}(\phi) \\
\geq & \lambda\left(\theta_{m}\right)+\gamma_{m} \frac{\epsilon^{2}}{2}+\varepsilon_{m}(\phi) .
\end{aligned}
$$

Since $\varepsilon_{m}(\phi)$ is summable (Lemma 4 ), but $\sum_{m} \gamma_{m}=\infty$, we conclude that $\lambda\left(\theta_{m}\right)$ converges to infinity, which is a contradiction.

Next we show that $\limsup _{m \rightarrow \infty}\left\|\nabla \lambda\left(\theta_{m}\right)\right\|=0$. Suppose the contrary. Then, there exists some $\epsilon>0$ such that $\left\|\nabla \lambda\left(\theta_{n}\right)\right\|>\epsilon$ for infinitely many indices $n$. For any such $n$, let $n^{\prime}$ be the first subsequent time that $\left\|\nabla \lambda\left(\theta_{n^{\prime}}\right)\right\|<\epsilon / 2$. Then,

$$
\begin{aligned}
\frac{\epsilon}{2} & \leq\left\|\nabla \lambda\left(\theta_{n}\right)\right\|-\left\|\nabla \lambda\left(\theta_{n^{\prime}}\right)\right\| \\
& \leq\left\|\nabla \lambda\left(\theta_{n}\right)-\nabla \lambda\left(\theta_{n^{\prime}}\right)\right\| \\
& \leq C\left\|r_{n}-r_{n^{\prime}}\right\| \\
& =C\left\|\sum_{m=n}^{n^{\prime}-1} \gamma_{m} h\left(r_{m}\right)+\sum_{m=n}^{n^{\prime}-1} \varepsilon_{m}\right\| \\
& \leq C \sum_{m=n}^{n^{\prime}-1} \gamma_{m}\left\|h\left(r_{m}\right)\right\|+C\left\|\sum_{m=n}^{n^{\prime}-1} \varepsilon_{m}\right\|,
\end{aligned}
$$

for some constant $C$, as $\nabla^{2} \lambda(\theta)$ is bounded (Lemma 1). Recall that $\left\|h\left(r_{m}\right)\right\|$ is bounded by some constant $B$. Furthermore, when $n$ is large enough, the summability of the sequence $\varepsilon_{m}$ yields $C\left\|\sum_{m=n}^{n^{\prime}-1} \varepsilon_{m}\right\| \leq \epsilon / 4$. This implies that $\sum_{m=n}^{n^{\prime}-1} \gamma_{m} \geq$ $\epsilon / 4 C B$. By an argument very similar to the one that led to (28), it is easily shown that there exists some $\beta>0$ such that

$$
\lambda\left(\theta_{n^{\prime}}\right) \geq \lambda\left(\theta_{n}\right)+\beta
$$

which contradicts the convergence of the sequence $\lambda\left(\theta_{m}\right)$.

\section{APPENDIX II}

\section{PROOF OF PROPOSITION 4}

In this section, we prove the convergence of the on-line method introduced in Section IV, which is described by

$$
\begin{aligned}
& \theta_{k+1}=\theta_{k}+\gamma_{k}\left(\nabla g_{i_{k}}\left(\theta_{k}\right)+\left(g_{i_{k}}\left(\theta_{k}\right)-\tilde{\lambda}_{k}\right) z_{k}\right) \\
& \tilde{\lambda}_{k+1}=\tilde{\lambda}_{k}+\eta \gamma_{k}\left(g_{i_{k}}\left(\theta_{k}\right)-\tilde{\lambda}_{k}\right), \\
& z_{k+1}= \begin{cases}0, & \text { if } i_{k+1}=i^{*} \\
z_{k}+\frac{\nabla p_{i_{k} i_{k+1}}\left(\theta_{k}\right)}{p_{i_{k} i_{k+1}}\left(\theta_{k}\right)}, & \text { otherwise. }\end{cases}
\end{aligned}
$$

The proof has many common elements with the proof of Proposition 3. For this reason, we will only discuss the differences in the two proofs. In addition, whenever routine arguments are used, we will only provide an outline.

As in Appendix I, we let $r_{k}=\left(\theta_{k}, \tilde{\lambda}_{k}\right)$. Note, however, the different meaning of the index $k$ which is now advanced at each time step, whereas in Appendix I it was advanced whenever the state $i^{*}$ was visited. We also define an augmented state $x_{k}=$ $\left(i_{k}, z_{k}\right)$.

We rewrite the update equations as

$$
r_{k+1}=r_{k}+\gamma_{k} R\left(x_{k}, r_{k}\right)
$$

where

$$
R\left(x_{k}, r_{k}\right)=\left[\begin{array}{c}
\nabla g_{i_{k}}\left(\theta_{k}\right)+\left(g_{i_{k}}\left(\theta_{k}\right)-\tilde{\lambda}_{k}\right) z_{k} \\
\eta\left(g_{i_{k}}\left(\theta_{k}\right)-\tilde{\lambda}_{k}\right)
\end{array}\right] .
$$

Consider the sequence of states $\left(i_{0}, i_{1}, \cdots\right)$ visited during the execution of the algorithm. As in Section III, we let $t_{m}$ be the $m$ th time that the recurrent state $i^{*}$ is visited. Also, as in Appendix I, we let

$$
\mathcal{F}_{m}=\left\{\theta_{0}, \tilde{\lambda}_{0}, i_{0}, \ldots, i_{t_{m}}\right\}
$$

stand for the history of the algorithm up to and including time $t_{m}$.

The parameter $\theta_{k}$ keeps changing between visits to state $i^{*}$, which is a situation somewhat different than that considered in Lemma 2a). Nevertheless, using Assumption 5, a similar argument applies and shows that for any positive integer $s$, there exists a constant $D_{s}$ such that

$$
E\left[\left(t_{m+1}-t_{m}\right)^{s} \mid \mathcal{F}_{m}\right] \leq D_{s}
$$

We have

$$
\begin{aligned}
r_{t_{m+1}} & =r_{t_{m}}+\sum_{k=t_{m}}^{t_{m+1}-1} \gamma_{k} R\left(x_{k}, r_{k}\right) \\
& =r_{t_{m}}+\tilde{\gamma}_{m} \hat{h}\left(r_{t_{m}}\right)+\varepsilon_{m}
\end{aligned}
$$

where $\tilde{\gamma}_{m}$ and $\varepsilon_{m}$ are given by

$$
\begin{aligned}
& \tilde{\gamma}_{m}=\sum_{k=t_{m}}^{t_{m+1}-1} \gamma_{k} \\
& \varepsilon_{m}=\sum_{k=t_{m}}^{t_{m+1}-1} \gamma_{k}\left(R\left(x_{k}, r_{k}\right)-\hat{h}\left(r_{t_{m}}\right)\right)
\end{aligned}
$$

and $\hat{h}$ is a scaled version of the function $h$ in Appendix I, namely,

$$
\hat{h}(r)=\frac{h(r)}{E_{\theta}[T]}=\left[\begin{array}{c}
\nabla \lambda(\theta)+\frac{G(\theta)}{E_{\theta}[T]}(\lambda(\theta)-\tilde{\lambda}) \\
\eta(\lambda(\theta)-\tilde{\lambda})
\end{array}\right] .
$$

We note the following property of the various stepsize parameters.

\section{Lemma 12:}

1) For any positive integer $s$, we have

$$
E\left[\sum_{m=1}^{\infty} \gamma_{t_{m}}^{2}\left(t_{m+1}-t_{m}\right)^{s}\right]<\infty .
$$


2) We have

$$
\sum_{m=1}^{\infty} \tilde{\gamma}_{m}=\infty, \quad \sum_{m=1}^{\infty} \tilde{\gamma}_{m}^{2}<\infty
$$

with probability 1 .

Proof:

1) From (30), and because $\gamma_{t_{m}}$ is $\mathcal{F}_{m}$-measurable, we have

$$
\begin{aligned}
E\left[\gamma_{t_{m}}^{2}\left(t_{m+1}-t_{m}\right)^{s}\right] & =E\left[\gamma_{t_{m}}^{2} E\left[\left(t_{m+1}-t_{m}\right)^{s} \mid \mathcal{F}_{m}\right]\right] \\
& \leq E\left[\gamma_{t_{m}}^{2}\right] D_{s} .
\end{aligned}
$$

Hence,

$$
\sum_{m=1}^{\infty} E\left[\gamma_{t_{m}}^{2}\left(t_{m+1}-t_{m}\right)^{s}\right] \leq D_{s} \sum_{k=1}^{\infty} \gamma_{k}^{2}<\infty
$$

and the result follows.

2) By Assumption 4, we have

$$
\sum_{m=1}^{\infty} \tilde{\gamma}_{m}=\sum_{k=1}^{\infty} \gamma_{k}=\infty
$$

Furthermore, since the sequence $\gamma_{k}$ is nonincreasing (Assumption 5), we have

$$
\tilde{\gamma}_{m}^{2} \leq \gamma_{t_{m}}^{2}\left(t_{m+1}-t_{m}\right)^{2}
$$

Using part a) of the lemma, we obtain that $\sum_{m=1}^{\infty} \tilde{\gamma}_{m}^{2}$ has finite expectation and is therefore finite with probability 1.

Without loss of generality, we assume that $\eta_{k} \leq 1$ for all $k$. Then, the update equation for $\tilde{\lambda}_{k}$ implies that $\left|\tilde{\lambda}_{k}\right| \leq$ $\max \left\{\left|\tilde{\lambda}_{0}\right|, C\right\}$, where $C$ is a bound on $\left|g_{i}(\theta)\right|$. Thus, $\left|\tilde{\lambda}_{k}\right|$ is bounded by a deterministic constant, which implies that the magnitude of $\hat{h}\left(r_{k}\right)$ is also bounded by a deterministic constant.

We now observe that (31) is of the same form as (25) that was studied in the preceding appendix, except that we now have $r_{t_{m}}$ in place of $r_{m}, \tilde{\gamma}_{m}$ in place of $\gamma_{m}$, and $\hat{h}\left(r_{t_{m}}\right)$ in place of $h\left(r_{m}\right)$. By Lemma 12b), the new stepsizes satisfy the same conditions as those imposed by Assumption 4 on the stepsizes $\gamma_{m}$ of Appendix I. Also, in the next subsection, we show that the series $\sum_{m} \varepsilon_{m}$ converges. Once these properties are established, the arguments in Appendix I remain valid and show that $\lambda\left(\theta_{t_{m}}\right)$ converges, and that $\nabla \lambda\left(\theta_{t_{m}}\right)$ converges to zero. Furthermore, we will see in the next subsection that the total change of $\theta_{k}$ between consecutive visits to $i^{*}$ converges to zero. This implies that $\lambda\left(\theta_{k}\right)$ converges and that $\nabla \lambda\left(\theta_{k}\right)$ converges to zero, and Proposition 4 is established.

\section{A. Summability of $\varepsilon_{k}$ and Convergence of the Changes in $\theta_{k}$}

This subsection is devoted to the proof that the series $\sum_{m} \varepsilon_{m}$ converges, and that the changes of $\theta_{k}$ between visits to $i^{*}$ converge to zero.

We introduce some more notation. The evolution of the augmented state $x_{k}=\left(i_{k}, z_{k}\right)$ is affected by the fact that $\theta_{k}$ changes at each time step. Given a time $t_{m}$ at which $i^{*}$ is visited, we define a "frozen" augmented state $x_{k}^{F}=\left(i_{k}^{F}, z_{k}^{F}\right)$ which evolves the same way as $x_{k}$ except that $\theta_{k}$ is held fixed at $\theta_{t_{m}}$ until the next visit at $i^{*}$. More precisely, we let $x_{t_{m}}^{F}=x_{t_{m}}$. Then, for $k \geq t_{m}+1$, we let $i_{k}^{F}$ evolve as a time-homogeneous
Markov chain with transition probabilities $p_{i j}\left(\theta_{t_{m}}\right)$. We also let $t_{m+1}^{F}=\min \left\{k>t_{m} \mid i_{k}^{F}=i^{*}\right\}$ be the first time after $t_{m}$ that $i_{k}^{F}$ is equal to $i^{*}$, and

$$
z_{k+1}^{F}=z_{k}^{F}+L_{i_{k}^{F} i_{k+1}^{F}}\left(\theta_{t_{m}}\right)
$$

We start by breaking down $\varepsilon_{m}$ as follows:

$$
\begin{aligned}
\varepsilon_{m} & =\sum_{k=t_{m}}^{t_{m+1}-1} \gamma_{k}\left(R\left(x_{k}, r_{k}\right)-\hat{h}\left(r_{t_{m}}\right)\right) \\
& =\varepsilon_{m}^{(1)}+\varepsilon_{m}^{(2)}+\varepsilon_{m}^{(3)}+\varepsilon_{m}^{(4)}+\varepsilon_{m}^{(5)}
\end{aligned}
$$

where

$$
\begin{aligned}
& \varepsilon_{m}^{(1)}=\sum_{k=t_{m}}^{t_{m+1}-1}\left(\gamma_{t_{m}}-\gamma_{k}\right) \hat{h}\left(r_{t_{m}}\right) \\
& \varepsilon_{m}^{(2)}=\gamma_{t_{m}} \sum_{k=t_{m}}^{t_{m+1}^{F}-1}\left[R\left(x_{k}^{F}, r_{t_{m}}\right)-\hat{h}\left(r_{t_{m}}\right)\right] \\
& \varepsilon_{m}^{(3)}=\gamma_{t_{m}} \sum_{k=t_{m}}^{t_{m+1}-1}\left[R\left(x_{k}, r_{t_{m}}\right)-\hat{h}\left(r_{t_{m}}\right)\right] \\
& \varepsilon_{m}^{(4)}=\gamma_{t_{m}} \sum_{k=t_{m}}^{t_{t_{m}}^{F}} \sum_{k=t_{m}}^{t_{m+1}-1}\left[R\left(x_{k}, r_{k}\right)-R\left(x_{k}, r_{t_{m}}\right)\right] \\
& \left.\varepsilon_{m}^{(5)}=\sum_{k=t_{m}}^{F}\left(\gamma_{k}-\gamma_{t_{m}}\right)-\hat{h}\left(r_{t_{m}}\right)\right]
\end{aligned}
$$

We will show that each one of the series $\sum_{m} \varepsilon_{m}^{(n)}, n=$ $1, \cdots, 5$, converges with probability 1 .

We make the following observations. The ratio $L_{i_{k} i_{k+1}}\left(\theta_{k}\right)$ is bounded because of Assumption 3. This implies that between the times $t_{m}$ and $t_{m+1}$ that $i^{*}$ is visited, the magnitude of $z_{k}$ is bounded by $C\left(t_{m+1}-t_{m}\right)$ for some constant $C$. Similarly, the magnitude of $z_{k}^{F}$ is bounded by $C\left(t_{m+1}^{F}-t_{m}\right)$. Using the boundedness of $\tilde{\lambda}_{k}$ and $\hat{h}\left(r_{k}\right)$, together with the update equations for $\theta_{k}$ and $\tilde{\lambda}_{k}$, we conclude that there exists a (deterministic) constant $C$, such that for every $m$, we have for $k \in$ $\left\{t_{m}, \ldots, t_{m+1}-1\right\}$

$$
\begin{aligned}
\left\|R\left(x_{k}, r_{k}\right)\right\| & \leq C\left(t_{m+1}-t_{m}\right) \\
\left\|r_{k}-r_{t_{m}}\right\| & \leq C \gamma_{t_{m}}\left(t_{m+1}-t_{m}\right)^{2} \\
\left\|R\left(x_{k}, r_{t_{m}}\right)-R\left(x_{k}, r_{k}\right)\right\| & \leq C \gamma_{t_{m}}\left(t_{m+1}-t_{m}\right)^{3}
\end{aligned}
$$

and for $k \in\left\{t_{m}, \ldots, t_{m+1}^{F}-1\right\}$

$$
\left\|R\left(x_{k}^{F}, r_{k}\right)\right\| \leq C\left(t_{m+1}^{F}-t_{m}\right) \text {. }
$$

Lemma 13: The series $\sum_{m} \varepsilon_{m}^{(1)}$ converges with probability 1.

Proof: Let $B$ be a bound on $\left\|\hat{h}\left(r_{k}\right)\right\|$. Then, using Assumption 5, we have

$$
\left\|\varepsilon_{m}^{(1)}\right\| \leq B \sum_{k=t_{m}}^{t_{m+1}-1}\left(\gamma_{t_{m}}-\gamma_{k}\right) \leq B A \gamma_{t_{m}}^{2}\left(t_{m+1}-t_{m}\right)^{p}
$$


Then, Lemma 12a) implies that $\sum_{m}\left\|\varepsilon_{m}^{(1)}\right\|$ has finite expectation, and is therefore finite with probability 1.

Lemma 14: The series $\sum_{m} \varepsilon_{m}^{(2)}$ converges with probability 1.

Proof: When the parameters $\theta$ and $\tilde{\lambda}$ are frozen to their values at time $t_{m}$, the total update $\sum_{k=t_{m}}^{t_{m+1}^{F}-1} R\left(x_{k}^{F}, r_{t_{m}}\right)$ coincides with the update $H_{m}\left(r_{m}\right)$ of the algorithm studied in Appendix I. Using the discussion in the beginning of that appendix, we have $E\left[H_{m}\left(r_{m}\right) \mid \mathcal{F}_{m}\right]=h\left(r_{t_{m}}\right)$. Furthermore, observe that

$$
E\left[\sum_{k=t_{m}}^{t_{m+1}^{F}-1} \hat{h}\left(r_{t_{m}}\right) \mid \mathcal{F}_{m}\right]=\hat{h}\left(r_{t_{m}}\right) E_{\theta_{t_{m}}}[T]=h\left(r_{t_{m}}\right) .
$$

Thus, $E\left[\varepsilon_{m}^{(2)} \mid \mathcal{F}_{m}\right]=0$. Furthermore, using (34), we have

$$
E\left[\left\|\varepsilon_{m}^{(2)}\right\|^{2} \mid \mathcal{F}_{m}\right] \leq C \gamma_{t_{m}}^{2}\left(t_{m+1}-t_{m}\right)^{4} .
$$

Using Lemma 12a), we obtain

$$
E\left[\sum_{m=1}^{\infty}\left\|\varepsilon_{m}^{(2)}\right\|^{2}\right]<\infty .
$$

Thus, $\sum_{m} \varepsilon_{m}^{(2)}$ is martingale with bounded variance and, therefore, converges.

Lemma 15: The series $\sum_{m} \varepsilon_{m}^{(3)}$ converges with probability 1.

Proof: The proof is based on a coupling argument. For $k=t_{m}, \ldots, t_{m+1}-1$, the two processes $x_{k}$ and $x_{k}^{F}$ can be defined on the same probability space as follows. Suppose that $i_{k}$ and $i_{k}^{F}$ are both equal to some particular state $i$. We partition the unit interval into $N$ subintervals, each of length $p_{i j}\left(\theta_{k}\right)$, $j=1, \cdots, N$. The next state $i_{k+1}$ is obtained by generating a uniform random variable $U$ and selecting the state $j$ associated with the particular subinterval into which $U$ belongs. The same random variable $U$ is used to select $i_{k+1}^{F}$, except that we now have a partition into subintervals of length $p_{i j}\left(\theta_{k}^{F}\right)$. The probability that $U$ causes $i_{k+1}$ and $i_{k+1}^{F}$ to be different is bounded by $N \max _{i, j}\left|p_{i j}\left(\theta_{k}\right)-p_{i j}\left(\theta_{k}^{F}\right)\right|$. Using the assumption that the transition probabilities depend smoothly on $\theta$, as well as (35), we obtain

$$
\begin{aligned}
P\left(i_{k+1}^{F} \neq i_{k+1} \mid i_{k}^{F}=i_{k}\right) & \leq B\left\|\theta_{k}-\theta_{k}^{F}\right\| \\
& \leq B\left\|r_{k}-r_{t_{m}}\right\| \\
& \leq B C \gamma_{t_{m}}\left(t_{m+1}-t_{m}\right)^{2}
\end{aligned}
$$

for some constants $B$ and $C$.

We define $\mathcal{E}_{m}$ to be the event

$$
\mathcal{E}_{m}=\left\{x_{k}^{F} \neq x_{k} \text { for some } k=t_{m}, \ldots, t_{m+1}\right\} .
$$

Using (38), we obtain

$$
\begin{aligned}
P\left(\mathcal{E}_{m} \mid t_{m}, t_{m+1}\right) & \leq B C \sum_{k=t_{m}}^{t_{m+1}-1} \gamma_{t_{m}}\left(t_{m+1}-t_{m}\right)^{2} \\
& =B C \gamma_{t_{m}}\left(t_{m+1}-t_{m}\right)^{3} .
\end{aligned}
$$

Note that, if the event $\mathcal{E}_{m}$ does not occur, then $\varepsilon_{m}^{(3)}=0$. Thus,

$$
\begin{aligned}
E\left[\left\|\varepsilon_{m}^{(3)}\right\| \mid t_{m}, t_{m+1}\right]= & P\left(\mathcal{E}_{m} \mid t_{m}, t_{m+1}\right) \\
& \times E\left[\left\|\varepsilon_{m}^{(3)}\right\| \mid t_{m}, t_{m+1}, \mathcal{E}_{m}\right] .
\end{aligned}
$$

Since $\hat{h}\left(r_{k}\right)$ is bounded, and using also the bounds (34)-(37), we have

$$
\left\|\varepsilon_{m}^{(3)}\right\| \leq \gamma_{t_{m}} C\left(\left(t_{m+1}-t_{m}\right)^{2}+\left(t_{m+1}^{F}-t_{m}\right)^{2}\right)
$$

for some new constant $C$. We conclude that

$$
\begin{aligned}
& E\left[\left\|\varepsilon_{m}^{(3)}\right\| \mid t_{m}, t_{m+1}, \mathcal{E}_{m}\right] \\
& \quad \leq \gamma_{t_{m}} C\left(t_{m+1}-t_{m}\right)^{2} \\
& \quad+\gamma_{t_{m}} C E\left[\left(t_{m+1}^{F}-t_{m}\right)^{2} \mid t_{m}, t_{m+1}, \mathcal{E}_{m}\right] .
\end{aligned}
$$

Now, it is easily verified that

$$
\begin{aligned}
& \left.E\left[\left(t_{m+1}^{F}-t_{m}\right)^{2} \mid t_{m}, t_{m+1}, \mathcal{E}_{m}\right]\right) \\
& \quad \leq 2 E\left[\left(t_{m+1}^{F}-t_{m+1}\right)^{2} \mid t_{m}, t_{m+1}, \mathcal{E}_{m}\right]+2\left(t_{m+1}-t_{m}\right)^{2} \\
& \quad \leq C\left(t_{m+1}-t_{m}\right)^{2}
\end{aligned}
$$

for some new constant $C$. By combining these inequalities, we obtain

and

$$
E\left[\left\|\varepsilon_{m}^{(3)}\right\| \mid t_{m}, t_{m+1}, \mathcal{E}_{m}\right] \leq C \gamma_{t_{m}}\left(t_{m+1}-t_{m}\right)^{2}
$$

$$
E\left[\left\|\varepsilon_{m}^{(3)}\right\||| t_{m}, t_{m+1}\right] \leq B C \gamma_{t_{m}}^{2}\left(t_{m+1}-t_{m}\right)^{5}
$$

for some different constant $C$. Using Lemma 12a), $\sum_{m}\left\|\varepsilon_{m}^{(3)}\right\|$ has finite expectation, and is, therefore, finite with probability 1.

Lemma 16: The series $\sum_{m} \varepsilon_{m}^{(4)}$ converges with probability 1.

Proof: Using (36), we have

$$
\begin{aligned}
\left\|\varepsilon_{m}^{(4)}\right\| & \leq \gamma_{t_{m}} \sum_{k=t_{m}}^{t_{m+1}-1} C \gamma_{t_{m}}\left(t_{m+1}-t_{m}\right)^{3} \\
& =C \gamma_{t_{m}}^{2}\left(t_{m+1}-t_{m}\right)^{4} .
\end{aligned}
$$

Using Lemma 12a), $\sum_{m}\left\|\varepsilon_{m}^{(4)}\right\|$ has finite expectation, and is therefore finite with probability 1 .

Lemma 17: The series $\sum_{m} \varepsilon_{m}^{(5)}$ converges with probability 1.

Proof: Using Assumption 5 and the bound (34) on $\left\|R\left(x_{k}, r_{k}\right)\right\|$, we have

$$
\begin{aligned}
\left\|\varepsilon_{m}^{(5)}\right\| & \leq C\left(t_{m+1}-t_{m}\right) \sum_{k=t_{m}}^{t_{m+1}-1}\left(\gamma_{t_{m}}-\gamma_{k}\right) \\
& \leq A C \gamma_{t_{m}}^{2}\left(t_{m+1}-t_{m}\right)^{p+1} .
\end{aligned}
$$

Using Lemma 12a), $\sum_{m}\left\|\varepsilon_{m}^{(5)}\right\|$ has finite expectation, and is, therefore, finite with probability 1 . 
We close by establishing the statement mentioned at the end of the preceding subsection, namely, that the changes in $r_{k}$ (and, therefore, the changes in $\theta_{k}$ as well) between visits to the recurrent state $i^{*}$ tend to zero as time goes to infinity. Indeed, (34) establishes a bound on $\left\|r_{k}-r_{t_{m}}\right\|$ for $k=t_{m}, \ldots, t_{m+1}-1$, which converges to zero because of Lemma 12a).

\section{ACKNOWLEDGMENT}

The authors are grateful to O. Mihatsch for suggesting the form of Assumption 3, and to the referees for their many suggestions on improving the paper.

\section{REFERENCES}

[1] D. P. Bertsekas, Dynamic Programming and Optimal Control. Belmont, MA: Athena, 1995.

[2] - Nonlinear Programming. Belmont, MA: Athena, 1995.

[3] V. S. Borkar, "Stochastic approximation with two time scales," Syst. Control Lett., vol. 29, no. 5, pp. 291-294, 1997.

[4] A. Barto, R. Sutton, and C. Anderson, "Neuron-like elements that can solve difficult learning control problems," IEEE Trans. Syst, Man, Cybern., vol. 13, pp. 835-846, Sept./Oct. 1983.

[5] D. P. Bertsekas and J. N. Tsitsiklis, Neuro-Dynamic Programming. Belmont, MA: Athena, 1996.

[6] - "Gradient convergence in gradient methods," Massachusetts Inst. Tech., Cambridge, MA, Lab. for Info. and Decision Systems Report LIDS-P-2301, 1997

[7] X. R. Cao and H. F. Chen, "Perturbation realization, potentials, and sensitivity analysis of Markov processes," IEEE Trans. Automat. Contr. vol. 42, pp. 1382-1393, Oct. 1997.

[8] E. K. P. Chong and P. J. Ramadage, "Stochastic optimization of regenerative systems using infinitesimal perturbation analysis," IEEE Trans. Automat. Contr., vol. 39, pp. 1400-1410, July 1994.

[9] X. R. Cao and Y. W. Wan, "Algorithms for sensitivity analysis of Markov systems through potentials and perturbation realization," IEEE Trans. Contr. Syst. Technol., vol. 6, pp. 482-494, July 1998.

[10] B. Delyon, "General results on the convergence of stochastic algorithms," IEEE Trans. Automat. Contr., vol. 41, pp. 1245-1255, Sept. 1996.

[11] M. C. Fu and J. Hu, "Smoothed perturbation analysis derivative estimation for Markov chains," Oper. Res. Lett., vol. 15, pp. 241-251, June 1994.

[12] P. Glasserman, Gradient Estimation Via Perturbation Analysis. Boston, MA: Kluwer, 1991.

[13] P. Glasserman and P. W. Glynn, "Gradient estimation for regenerative processes," in Proc. Winter Simulation Conf., 1992, pp. 280-288.

[14] P. W. Glynn, "Stochastic approximation for Monte Carlo optimization," in Proc. Winter Simulation Conf., 1986, pp. 285-289.

[15] — - "Likelihood ratio gradient estimation: An overview," in Proc. Winter Simulation Conf., 1987, pp. 366-375.

[16] P. W. Glynn and D. L. Iglehart, "Importance sampling for stochastic simulation," Manage. Sci., vol. 35, no. 11, pp. 1367-1392, 1989.

[17] Y. C. Ho and X. R. Cao, Perturbation Analysis of Discrete Event Systems. Boston, MA: KluwerPublisher, 1991.

[18] T. Jaakkola, S. P. Singh, and M. I. Jordan, "Reinforcement learning algorithm for partially observable Markov decision problems," in $A d-$ vances in Neural Information Processing Systems. San Francisco, CA: Morgan Kaufman, 1995, vol. 7, pp. 345-352.

[19] V. R. Konda and V. S. Borkar, "Actor-critic like learning algorithms for Markov Decision processes," SIAM J. Control Optim., vol. 38, no. 1, pp. 94-123, 1999.

[20] V. R. Konda and J. N. Tsitsiklis, "Actor-critic algorithms," in Proc. 1999 Neural Information Processing Systems Conf., to be published.

[21] P. L'Ecuyer, "A unified view of the IPA, SF, and LR gradient estimation techniques," Manage. Sci., vol. 36, no. 11, pp. 1364-1383, 1990.
[22] L. Ljung, "Analysis of recursive stochastic algorithms," IEEE Trans. Automat. Contr, vol. AC-22, pp. 551-575, Aug. 1977.

[23] P. Marbach, "Simulation-based optimization of Markov decision processes," Ph.D. dissertation, Dept. of Electr. Eng. Comp. Sci., Massachusetts Inst. Tech., Cambridge, MA, 1998.

[24] P. Marbach and J. N. Tsitsiklis, "Simulation-based optimization of Markov reward processes: Implementation issues," in Proc. IEEE Conf. Decision Control, Phoenix, AZ, December 1999.

[25] M. L. Puterman, Markov Decision Processes: Discrete Stochastic Dy namic Programming. New York: Wiley, 1994.

[26] R. S. Sutton and A. G. Barto, Reinforcement Learning: An Introduc tion. Cambridge, MA: MIT Press, 1998.

[27] R. S. Sutton, D. McAllester, S. Singh, and Y. Mansour, "Policy gradien methods for reinforcement learning with function approximation," in Proc. 1999 Neural Information Processing Systems Conf., to be published.

[28] G. J. Tesauro, "Practical issues in temporal difference learning," Mach. Learn., vol. 8, pp. 257-277, May 1992

[29] V. Tresp and R. Hofmann, "Missing and noisy data in nonlinear time-series prediction," in Neural Networks for Signal Processing, S. F. Girosi, J. Mahoul, E. Manolakos, and E. Wilson, Eds. New York: IEEE Press, 1995, pp. 1-10.

[30] C. Watkins, "Learning from delayed rewards," Ph.D. dissertation, Dept. of Psychology, Cambridge University, U.K., 1989.

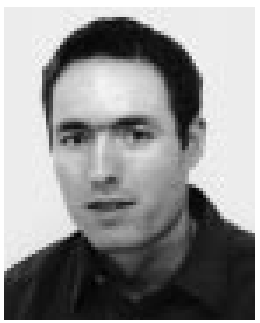

Peter Marbach was born in Lucerne, Switzerland. He received the Eidg. Dipl. El.-Ing. degree from the ETH Zurich, Switzerland, in 1993, the M.S. degree in electrical engineering from the Columbia University, NY in 1994, and the Ph.D. degree in electrical engineering from the Massachusetts Institute of Technology, Cambridge, MA, in 1998.

He was a Visiting Scientist at the Siemens Corporate Research Center, Munich Germany, in 1996. He was an Alcatel-Bell Post Doctoral Research Fellow with the Centre for Communication Systems Research at the University of Cambridge, U.K. Since 2000, he has been an assistant professor with the Computer Science Department at the University of Toronto, Toronto, Canada. His research interests are in the fields of communication networks, stochastic systems, optimization, and control.

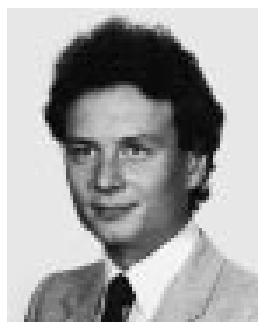

John N. Tsitsiklis (S'81-M'83-SM'97-F'99) was born in Thessaloniki, Greece, in 1958. He received the B.S. degree in mathematics, and the B.S., M.S., and $\mathrm{Ph} . \mathrm{D}$. degrees in electrical engineering, all from the Massachusetts Institute of Technology, Cambridge, in 1980, 1980, 1981, and 1984, respectively.

During the academic year 1983-1984, he was an Acting Assistant Professor of Electrical Engineering at Stanford University, Stanford, CA. Since 1984, he has been with the Massachusetts Institute of Technology, where he is currently Professor of Electrical Engineering and Computer Science. His research interests are in the fields of systems, optimization, control, and operations research. He is a coauthor of Parallel and Distributed Computation: Numerical Methods (Belmont, MA: Athena, 1989), Neuro-Dynamic Programming(Belmont, MA: Athena, 1996), and Introduction to Linear Optimization(Belmont, MA: Athena, 1997), all with D. Bertsimas.

Dr. Tsitsiklis was a recipient of an IBM Faculty Development Award in1983, an National Science Foundation Presidential Young Investigator Award in 1986, an Outstanding Paper Award by the IEEE Control Systems Society, the M.I.T. Edgerton Faculty Achievement Award in 1989, the Bodossakis Foundation Prize in1995), and the INFORMS/CSTS prize in1997. 